University of Redlands

\title{
Solid Waste Collection Vehicle Route Optimization for the City of Redlands, California
}

A Major Individual Project submitted in partial satisfaction of the requirements

for the degree of Master of Science in Geographic Information Systems

by

Dene L. O’Connor

Mark Kumler, Ph.D., Committee Chair

Douglas M. Flewelling, Ph.D.

Fang Ren, Ph.D.,

August 2013 
Solid Waste Collection Vehicle Route Optimization for the City of Redlands, California

Copyright (C) 2013

by

Dene L. O'Connor 
The report of Dene L. O'Connor is approved.
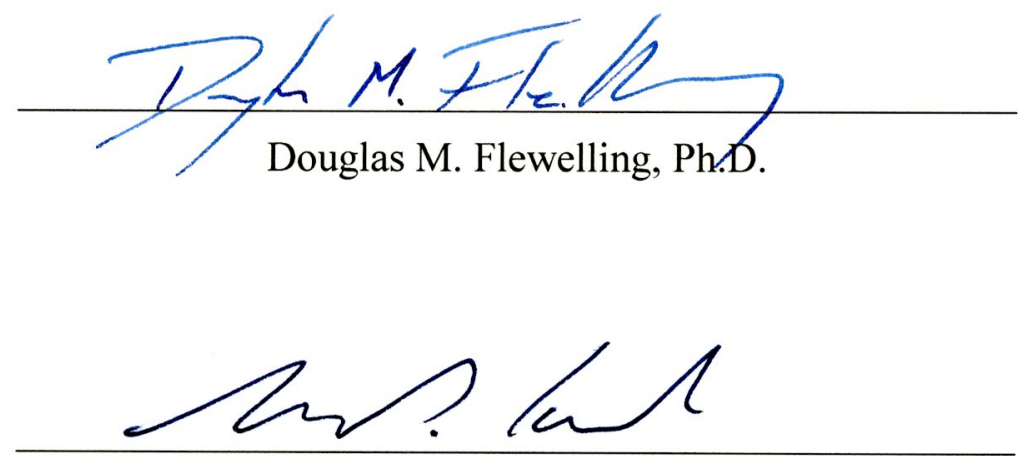

Mark Kumler, Ph.D., Committee Chair

August 2013 



\section{Acknowledgements}

I express appreciation to the City of Redlands, California for suggesting this project to the University of Redlands Master of Science GIS program. The City of Redlands GIS Supervisor Tom Resh and his staff were very helpful and responsive to any requests throughout the year. Thank you to all the Esri instructors who shared their knowledge, expertise and real world GIS experiences with our cohort. Very special thanks to Dr. Jay Sandhu from Esri. His extensive knowledge of GIS and Network Analyst expertise helped contribute to the success of this project. Dr. Sandhu was always very patient, responsive and accessible whenever I had questions or issues specific to the ArcGIS Network Analyst software extension. Thanks to the entire faculty at the University of Redlands MS GIS program. Special thanks to my advisor Dr. Fang Ren for her support and guidance in the early stages of the project and thanks to Dr. Mark Kumler for stepping in to help support the project's completion.

Cohort 22, thanks for all the good times, great memories and long study sessions together. I'd like to thank my family, friends and girlfriend for all their support and encouragement throughout a challenging year. 



\begin{abstract}
Solid Waste Collection Vehicle Route Optimization for the City of Redlands, California by

Dene L. O’Connor

The City of Redlands, California was interested in using a geographic information system (GIS) to help determine cost savings for the collection and transportation of its solid waste. Studies have shown that $60 \%-80 \%$ of a municipality's waste budget goes towards the collection and transportation phase. The city maintains a GIS department and they would like to incorporate data, procedures and a workflow to help facilitate using GIS to optimize solid waste collection. GIS technology can be used to help determine optimal collection routes by matching real world travel conditions and patterns. This study used a GIS to model current and proposed collection patterns using Esri's ArcGIS Network Analyst software. The software was used to determine optimal routes for small collection groups and outlines the workflow and best practices for future analysis throughout the city.
\end{abstract}





\section{Table of Contents}

Chapter 1 - Introduction ................................................................................................ 1

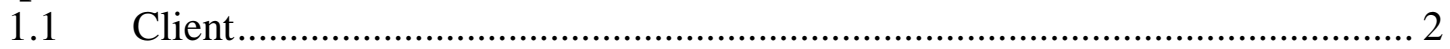

$1.2 \quad$ Problem Statement .......................................................................... 2

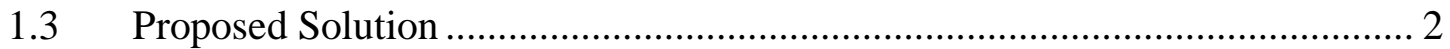

1.3.1 Goals and Objectives .......................................................................... 2

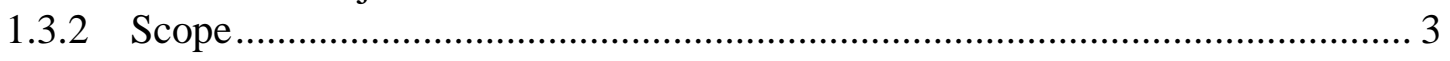

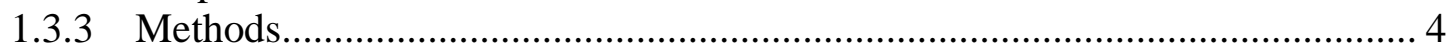

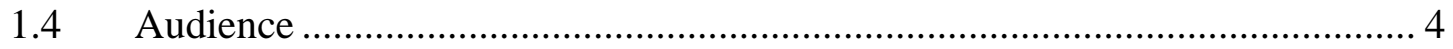

1.5 Overview of the Rest of this Report ......................................................... 4

Chapter 2 - Background and Literature Review ...........................................................5

$2.1 \quad$ Methods Used to Optimize Solid Waste Collection .......................................... 5

2.1.1 Route Optimization Using ArcGIS Network Analyst Software Extension ...... 5

2.1.1 Vehicle Routing Problem Solver in ArcGIS.................................................. 7

2.2 Applications of GIS in Waste Management ................................................ 7

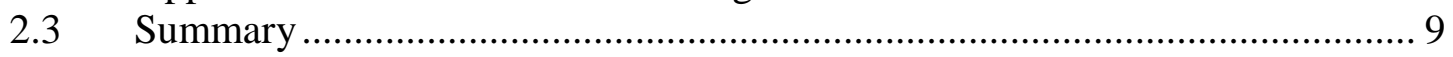

Chapter 3 - Systems Analysis and Design...................................................................... 11

$3.1 \quad$ Problem Statement .............................................................................. 11

3.2 Requirements Analysis ............................................................................. 11

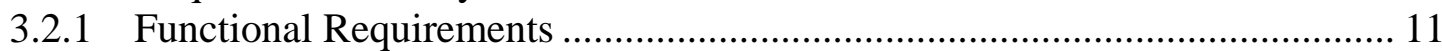

3.2.2 Non-Functional Requirements ........................................................... 12

3.3 System Design ................................................................................. 14

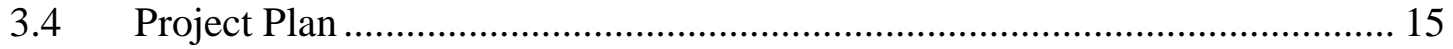

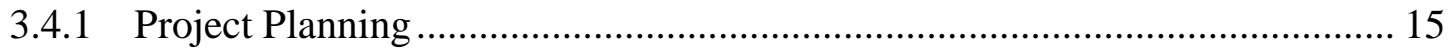

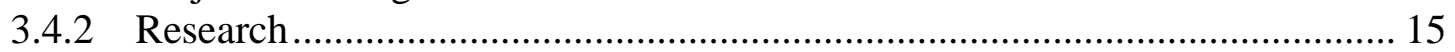

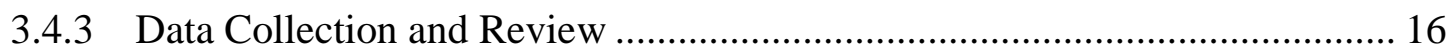

3.4.4 Project Implementation and Analysis ........................................................ 16

3.4.5 Project Finalization and Delivery ............................................................. 16

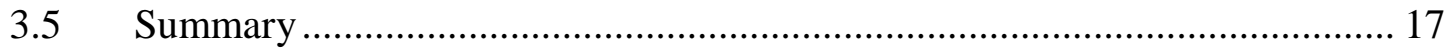

Chapter 4 - Database Design....................................................................................................... 19

4.1 Conceptual Data Model ......................................................................... 19

4.2 Logical Data Model …………………………................................ 20

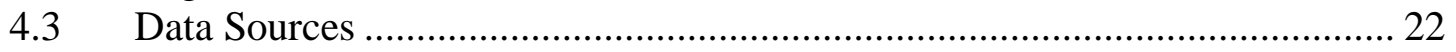

4.4 Data Collection Methods ........................................................................ 23

4.5 Data Scrubbing and Loading …………………....................................... 23

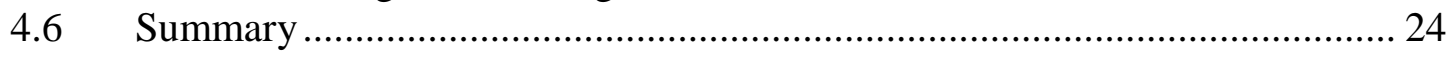

Chapter 5 - Implementation.............................................................................................. 25

$5.1 \quad$ Road Slope Analysis Experiment ……………………………………... 25

5.2 Road Network Dataset for Route Analysis ..................................................... 27

5.3 Vehicle Routing Problem Solver for Route Optimization................................ 31

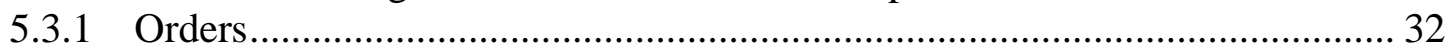

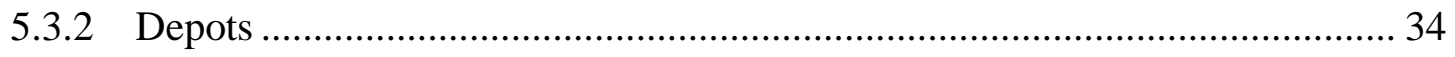

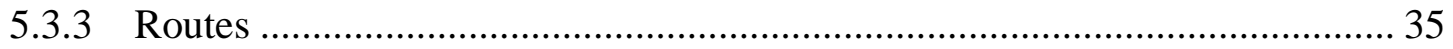




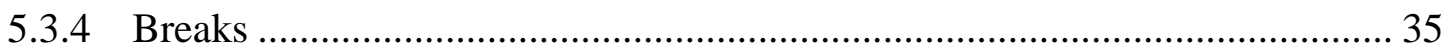

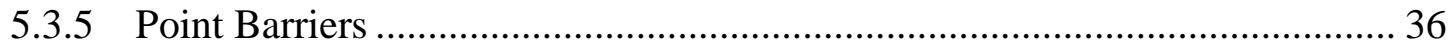

5.4 Executing the Vehicle Routing Problem Solver for New Routes .................... 37

5.5 Configuring Existing Collection Routes for VRP Solver ................................ 37

5.6 Various Input Versions for VRP Solver ..................................................... 38

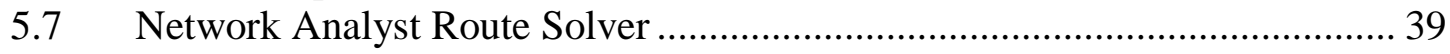

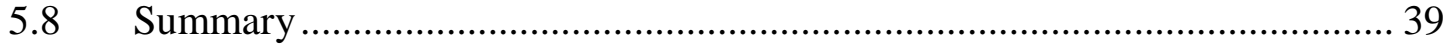

Chapter 6 - Results and Analysis............................................................................ 41

6.1 New Routes from the Network Analyst VRP Solver....................................... 41

6.2 Existing Routes from Network Analyst VRP Solver....................................... 44

6.3 Results from Network Analyst Route Solver.................................................. 46

6.4 How the City of Redlands Might Use the Results .......................................... 46

Chapter 7 - Conclusions and Future Work .................................................................. 49

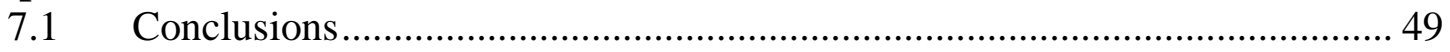

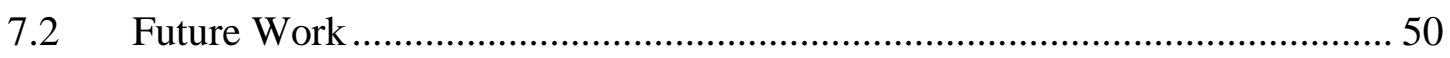

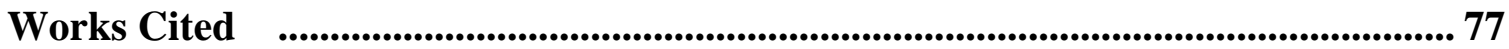




\section{Table of Figures}

Figure 1-1: The Study Location ................................................................................ 1

Figure 1-2: Redlands Solid Waste Collection Districts ......................................... 3

Figure 4-1: Conceptual Data Design Model ............................................................... 20

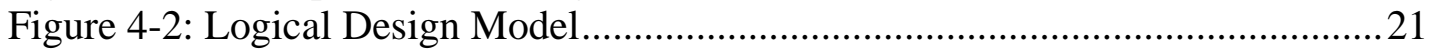

Figure 5-1: Network Analyst Vehicle Routing Problem Solver Workflow ..............25

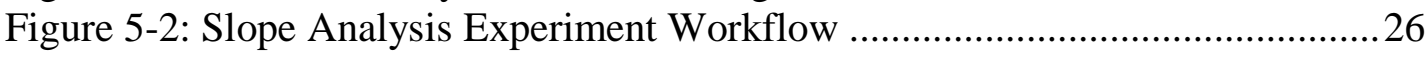

Figure 5-3: Network Dataset Construction Diagram .................................................28

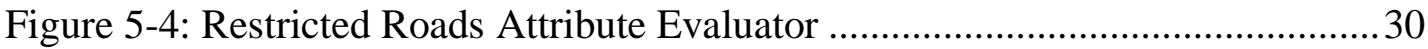

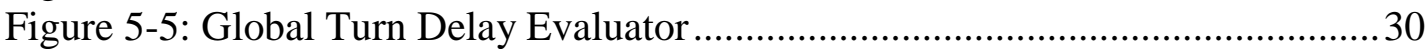

Figure 5-6: Vehicle Routing Problem Solver Detailed Workflow .............................. 32

Figure 5-8: Incorrect Order Location along the Network Dataset ............................... 34

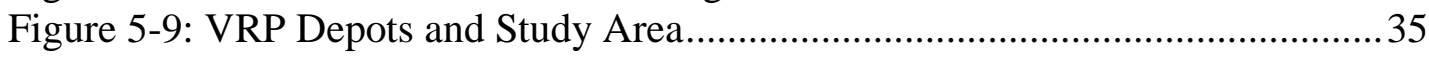

Figure 5-10: Added Cost Point Barriers .................................................................. 37

Figure 5-11: Network Analysis Vehicle Routing Problem Solver Layer Properties. 37

Figure 6-1: Anomalous Sequencing Results ..................................................... 42

Figure 6-2: Existing Route Collection Sequence Comparison ...............................45 



\section{List of Tables}

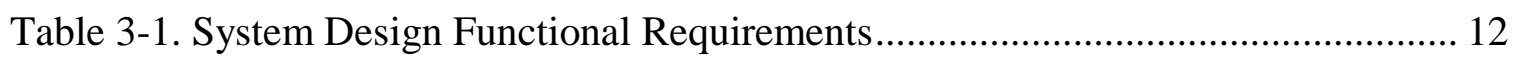

Table 3-2. System Design Non-Functional Requirements .......................................... 13

Table 6-1. Results Comparison Solving All 829 Collection Points................................. 43

Table 6-2. Results Comparison Solving 87 Collection Points........................................ 44

Table 6-3. Results from Network Analyst Route Solver ............................................. 46 



\section{List of Acronyms and Definitions}

$\begin{array}{ll}\text { 3D } & \text { Three Dimensional } \\ \text { 2D } & \text { Two Dimensional } \\ \text { CPU } & \text { Central Processing Unit } \\ \text { DTM } & \text { Digital Terrain Model } \\ \text { GB } & \text { Gigabyte } \\ \text { GPS } & \text { Global Positioning System } \\ \text { MIN } & \text { Minutes } \\ \text { MSW } & \text { Municipal Solid Waste } \\ \text { NA } & \text { Network Analyst } \\ \text { PC } & \text { Personal Computer } \\ \text { QOL } & \text { Quality of Life } \\ \text { RAM } & \text { Random Access Memory } \\ \text { SWMP } & \text { Solid Waste Management Program } \\ \text { UML } & \text { Unified Modeling Language } \\ \text { VRP } & \text { Vehicle Routing Problem }\end{array}$





\section{Chapter 1 - Introduction}

The typical municipal garbage truck travels approximately 25,000 miles annually, gets less than three miles per gallon, and uses around 8,600 gallons of fuel per year (Cannon, 2005). With four California cities already filing for bankruptcy in 2012, being fiscally responsible and looking for cost saving measures should be a top priority for many cities. The City of Redlands wanted to take a proactive approach and looked for solutions.

Redlands is located in San Bernardino County, 60 miles east of downtown Los Angeles in a region called the Inland Empire (Figure 1-1). The city covers 36 square miles and has a population of 68,747 (CensusViewer, 2013). Redlands is divided into 23 sanitation collection districts. The city's sanitation fleet consists of 28 vehicles, servicing 19,000 residential and 1,800 commercial customers six days a week. Studies have shown up to $75 \%$ of a sanitation department's budget goes directly to solid waste collection and transport. This is clearly an area for city officials to evaluate for cost saving measures. Therefore, the city's Quality of Life Department was looking for more efficient truck routes to reduce its overall costs for collecting municipal solid waste from residential homes.

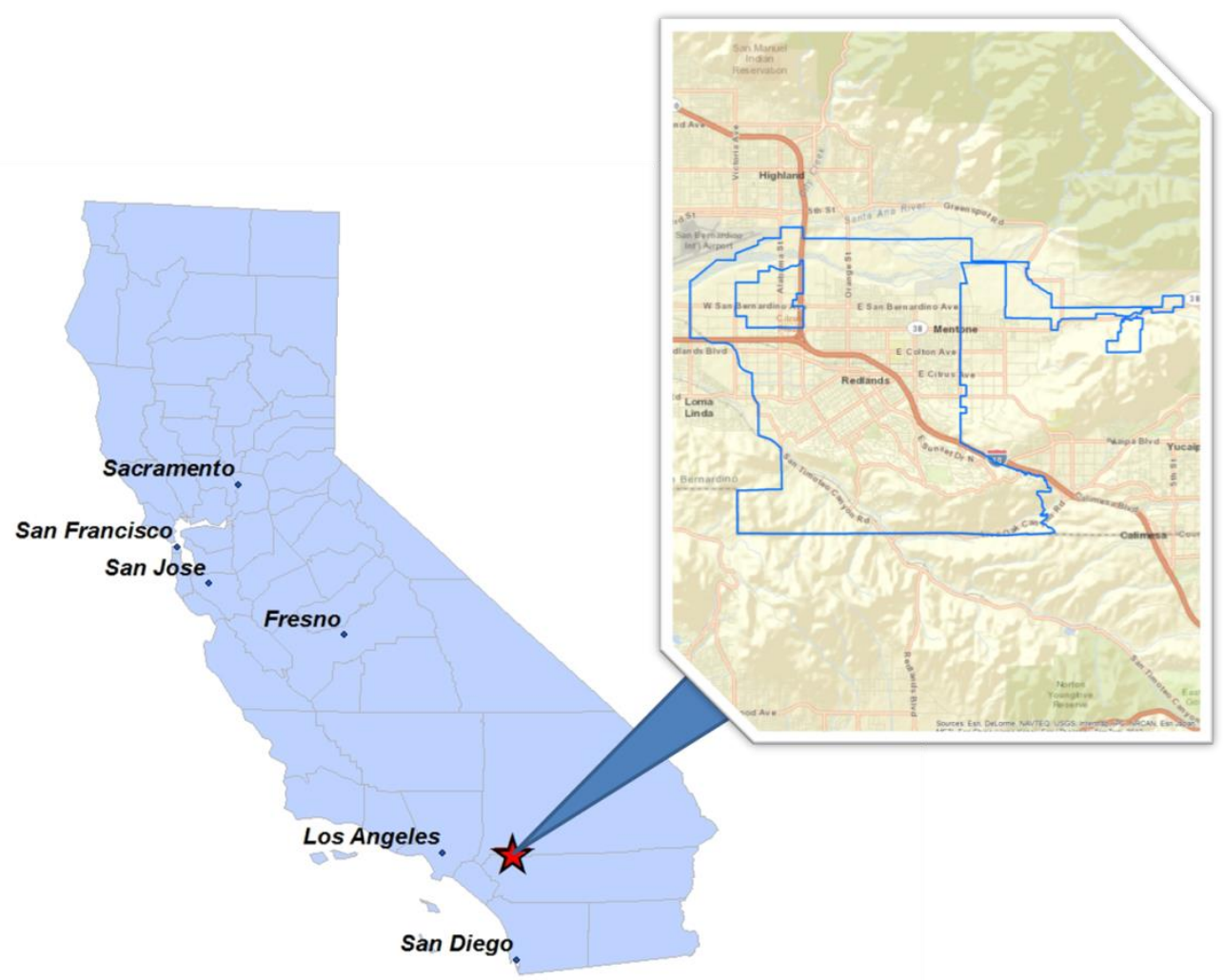

Figure -1: The Study Location 
Collaborating with the city's GIS Department, a Vehicle Routing Problem (VRP) optimization study was conducted to minimize the total travel time required for solid waste collection from residential homes. The vehicle routing solver provided in ArcGIS was applied to calculate optimized collection sequencing and routes. The project also compared current and new routes and discussed several issues that occurred in the analysis, which will provide helpful insight into future use of the software for tackling similar problems.

\subsection{Client}

The client for the project was the City of Redlands, California. The project contact was Tom Resh, GIS Supervisor/Web Administrator for the City of Redlands. The client wanted a geographic information system designed to calculate more efficient routes for sanitation collection and transport.

\subsection{Problem Statement}

The typical municipal garbage truck travels approximately 25,000 miles annually, gets less than three miles per gallon, and uses around 8,600 gallons of fuel per year (Cannon, 2005). A large part of a city sanitation budget goes towards fuel, maintenance, and labor for its fleet of sanitation collection vehicles. The City of Redlands wanted to use GIS technology to help reduce overall costs. The city was not sure how to design better travel routes to enhance efficiency, as it is a complex problem involving many factors, including the location of waste bins, collection details, operational hours, driving habits, type of vehicle, travel impedances, and integrity of road network being traversed. The city wanted to know the best approach and configurations using a GIS to help optimize sanitation collection routing.

\subsection{Proposed Solution}

This project aimed to produce optimized routes for sanitation trucks in a select study area of the city. Esri Network Analyst extension to ArcGIS was used to calculate the most efficient routes for the fleet vehicles to take, focusing on reducing travel drive time and overall miles driven. This program is the client's preferred software used to calculate point-to-point routing optimization. The Network Analyst extension uses complex algorithms to calculate cost or effort for travel over a connected network of roads. Some of the parameters considered in the program include road segment length, vehicle speed, break time, vehicle capacity, number of turns taken, traffic volume, and the number of stops signs and traffic lights. Using this program, new routes were calculated, examined, and compared to the existing collection routes.

\subsubsection{Goals and Objectives}

The primary goal of this project was to reduce overall travel cost for collection and transport of municipal solid waste from residential homes within Redlands. 
The project objectives were:

- Reduce the overall amount of miles driven to collect and transport residential waste container bins.

- Lessen the overall vehicle drive time to collect and transport residential waste container bins.

- Provide a template to use for optimizing waste collection and transport on remaining sanitation districts.

\subsubsection{Scope}

The scope of the project was to develop a detailed road network dataset for the study area, which includes stop signs and traffic lights. This involved exploring how different settings for the VRP parameters affect the optimization results. The results from the new routes were examined and compared to existing routes. While the City of Redlands is divided into 23 sanitation collection districts, this analysis focused on one collection district (Figure 1-2).

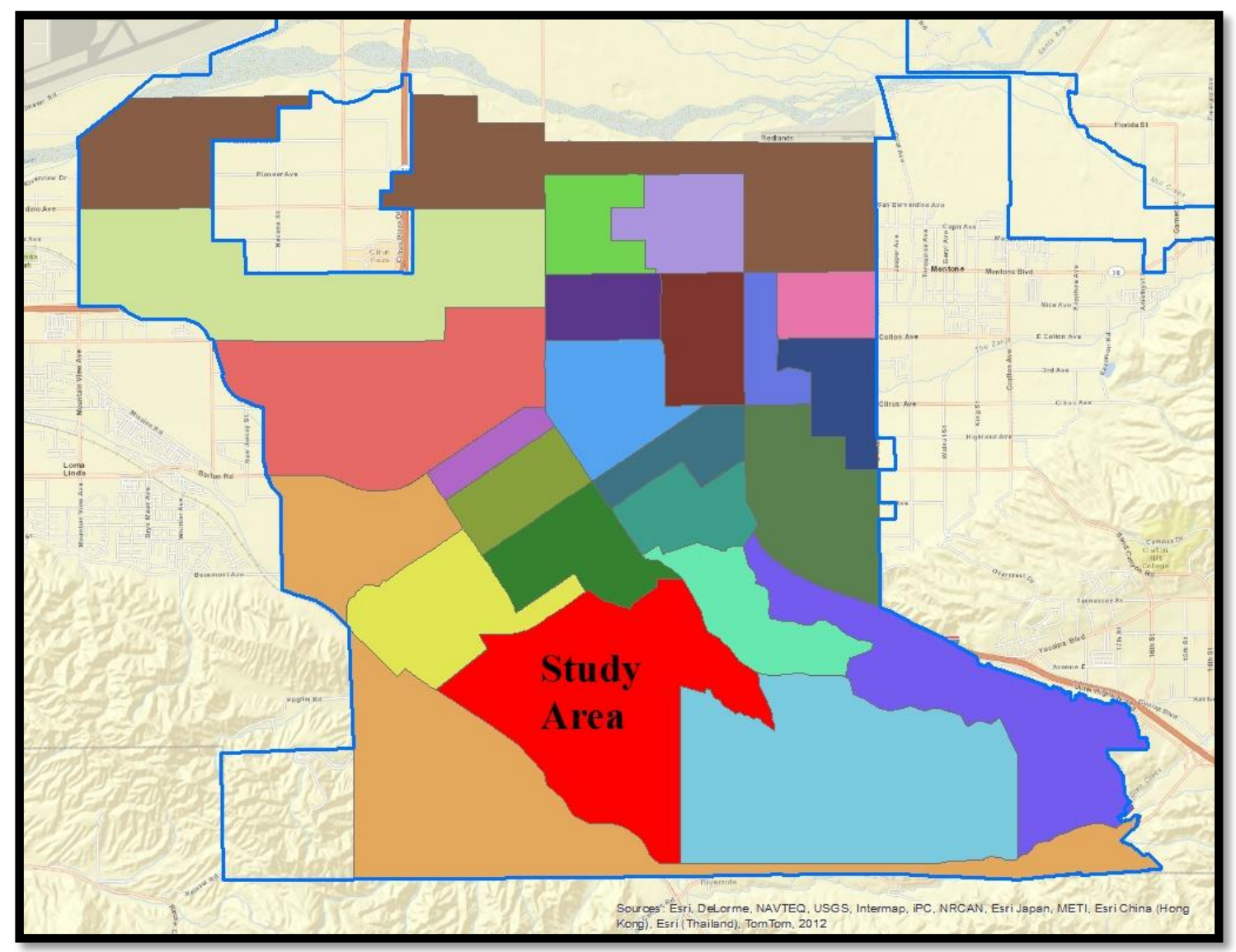

Figure 1-2: Redlands Solid Waste Collection Districts 


\subsubsection{Methods}

The software needed for this project was Esri's ArcGIS Desktop version 10.1 with the Network Analyst (NA) extension enabled. Network Analyst Vehicle Routing Problem solver was used to perform route calculations.

A waterfall project management approach was used for this study. The major steps for this project were as follows: the planning phase of the project involved discussions with the client and identifying the requirements analysis. The research phase involved inquiries into similar case studies specific to using a GIS for solid waste management and route optimization. The data collection phase involved discussions and meetings with the client to determine availability of existing data and what data needed to be edited and constructed from scratch. Implementation phase required configuring the data, different network parameters, executing the solver analysis, and repeating the steps as needed until desired results were achieved. The finalization phase included reviewing the results, documenting the procedures, creating final deliverables, then discussing and transfering them to the client.

\subsection{Audience}

This report discusses the procedures used in a GIS environment and is intended for GIS professionals with knowledge of ArcGIS software. The target audience should have an understanding of the Network Analyst extension Vehicle Routing Problem solver. This gives the reader a better understanding of the terms, procedures, and techniques used throughout the route planning analysis. Municipal workers, sanitation officials, and fleet vehicle personnel may find interest in the overall concept and benefits of the project.

\subsection{Overview of the Rest of this Report}

The balance of this report discusses the project components and how they were applied. They consist of chapters Two through Seven. Chapter Two describes the background and literature review, including information from previous studies done on route optimization for solid waste collection. Chapter Three explains the systems analysis and design phases of the project and identifies the client's problem and the design plan constructed to address the problem. Chapter Four defines the database design and structure the project used including, the data model used, data sources, and loading of datasets. Chapter Five refers to the implementation and the procedures taken to complete the project and provides detailed insight on how to build a similar type model. Chapter Six provides the results and a breakdown of the complete project and describes the lessons learned, what aspects were a success, which were unsuccessful, and identifies areas for future improvement. Chapter Seven discusses the overall conclusions of the project and future work that could potentially be expanded upon from this project. 


\section{Chapter 2 - Background and Literature Review}

There is an increasing awareness and need for cities and owners of fleet vehicles to reduce their overall operational costs. More and more cities continue to look for any cost saving measures available. The use of GIS technology has been one aspect that cost conscious decision makers implement to keep costs down. This is because GIS provides a powerful resource for identifying cost savings with "where" features are located geospatially, and how to travel to them more efficiently. This chapter reviews the past studies on how municipalities can optimize the collection of municipal solid waste (MSW), what current technologies are available, and how they are used.

\subsection{Methods Used to Optimize Solid Waste Collection}

"It has been estimated that, of the total amount of money spent for the collection, transportation, and the disposal of solid waste, approximately $60-80 \%$ is spent on the collection phase" (Karadimas, Doukas, Kolokathi, \& Defteraiou, 2008, p. 2022). This provides great opportunity for research to be conducted and to find better cost saving measures for municipalities. In addition to high costs to operate and maintain municipal vehicles, there is concern that sanitation trucks have a negative impact on the environment due to the quantity of miles driven, fuel type, engine inefficiency, and exhaust gases emissions. Solid waste management comprises the generation, collection, transport, treatment, and disposal of solid waste from a facility (Modak, 1996). The routing optimization problem in waste management has typically been addressed with different types of mathematical algorithms. Routing algorithms use a measuring system called a path length to determine the ideal route to a defined destination. The optimal routes are then determined by comparing the different paths. These paths can be calculated by different types of algorithms. Some of the routing algorithms used include Simulated Annealing, Tabu Search, Genetic Algorithm, Ant Colony Optimization, and Dijkstra's algorithm (Karadimas, Kolokathi, Defteraiou, \& Loumas, 2007).

\subsubsection{Route Optimization Using ArcGIS Network Analyst Software Extension}

Esri's ArcGIS Network Analyst extension allows users to perform complex calculations to solve vehicle routing problems. The program performs analysis over a network of connected edges and decides fleet routing, travel directions, closest facility, service area, and location allocation. In the application for route optimization, network dataset edges represent the road network being traversed. Network Analyst allows the user to dynamically model genuine network situations. These conditions can include speed limit, traffic volume at different times of the day, one-way streets, turn restrictions, obstacles, road conditions, and limitations.

Network Analyst key functions include:

- Establish a network with current GIS data.

- Detect closest facilities.

- Produce travel network cost matrix. 
- Define optimal facility positions using location allocation.

- Use time windows in vehicle scheduling.

- Determine shortest routes to travel.

Network Analyst mainly uses Dijkstra's algorithm, which is a simpler algorithm that finds the shortest or lowest cost path between two points. This algorithm preserves balance between evaluating a near optimal path to travel with one that is computationally practical. Dijkstra's algorithm divides the network dataset into lines or edges, with each edge representing a traversable or non-traversable piece of the network. In addition, each network edge also has an associated cost which represents the effort to travel that specific segment of road. These costs are calculated using one of two different criteria. The distance criterion is based on total edge length, and the time criterion measures edge length and time to traverse a segment (Karadimas, Doukas, Kolokathi, \& Defteraiou, 2008). The algorithm creates nodes or junctions at the start, end, and intersection of all edges; this defines the network by confirming there is transitivity between edges and junctions through the entire connected road network. The software calculates the cost to reach a node then determines the least cost path to travel to the next node. This continues until a final destination point is reached. These steps create only a temporary and partial solution. Once the initial cost is calculated between all the stops, the software applies a Tabu-Search heuristic process. This reevaluates and confirms, then reestablishes a more optimal path. This procedure continually runs to optimize the current path until no further optimization can be performed. The result is the least cost route to travel, along a path from a start to end point. Depending on number of points (stops) to make and complexity of the network, the analysis can take seconds or hours to complete. Karadimas, et al. (2008) reported that using Network Analyst route optimization for waste bin collection in 
Athens, Greece, yielded a 30\% decrease in the amount of communal bins needed within the city. Reducing the quantity from 162 to 112 waste bins produced a substantial savings in energy used for waste collection.

\subsubsection{Vehicle Routing Problem Solver in ArcGIS}

The Vehicle Routing Problem (VRP) solver is a component of Esri's ArcGIS Network Analyst software extension. The VRP calculates the least cost path for a fleet of vehicles to service one or more stops to minimize overall operating cost. Setting time windows in the VRP designates the beginning and end times allowed to service an item. This forces the solver to calculate the route to complete within that time frame; if it cannot achieve that an alert message is displayed. The VRP provides allotment for driver break periods, which is another feature to closely model true conditions. The VRP solver can assign capacity values to each route; this controls the instance, volume, weight, or quantity a vehicle can accept or deliver for each route. Specialty criteria can be configured for each order or route, this enables the solver to match specialties to certain orders. An example would be, a landscape company can only have properly licensed technicians apply certain lawn pesticides; the solver would match the order and route based on the specialty criteria setting. The VRP route zone feature delineates work territories for given routes and is used to constrain routes to servicing only those orders that fall within or near the specified area. An example would be some employees don't have the required permits to perform work in certain states or communities; the solver would exclude those drivers from the analysis. Another nice feature of the VRP is it allows driving directions to be printed out or exported from any of the routes being solved. The VRP solver was introduced with the ArcGIS version 9.3 software release. It allows users to apply specific network dataset rules that help solve the transportation problem. Many rules can be associated with real life waste collection particulars such as: fleet vehicle quantity, vehicle collection capacity, traffic conditions, work shift duration, service time to collect waste bins, and variation of waste production. The VRP solver is a relatively new feature with minimal literature available on case studies specific to waste collection and transport. The VRP solver is a significant element to consider whenever developing a waste collection route optimization plan (Jovicic, et al., 2011).

\subsection{Applications of GIS in Waste Management}

A key focus for any municipality is on introducing cost saving measures to keep departments within an annual budget. To achieve this goal, GIS technology has been applied to simplify some procedures. For example, (Karadimas, et. Al., 2007) used Esri's Network Analyst to create an optimized route to collect 15 bulk items from different locations within Attica, Greece, one of the 30 waste collection districts within Athens, Greece, covering $1.34 \mathrm{~km}^{2}$ with a population over 20,000 people. Athens produces roughly 5,000 tons of garbage each year. Bulk items consisted of oversized objects that standard garbage trucks could not remove such as furniture, large appliances, debris, and construction material. A geodatabase was created in ArcGIS that included the following 
data: bulk item locations, road networks, traffic volume, and population density. Network Analyst parameter settings were configured to calculate shortest distance and nighttime collection. The Network Analyst restrictions included street directions (one-way), no Uturns except dead-ends, displaying routes true shape. The current collection route was devised empirically and covered a distance of $5.7 \mathrm{~km}$. The newly optimized Network Analyst route covered $4.5 \mathrm{~km}$, for a $20 \%$ drop in kilometers driven to collect the items.

In another study (Jovicic, et al., 2011), ArcGIS Network Analyst was employed to calculate the shortest solid waste collection route, with the goal to reduce overall fuel costs, for City of Kragujevac, Serbia, which has approximately 4,000 waste bins at 2,000 locations within 12 city collection districts. The research considered one day shift collection truck, servicing a subsection area about $5 \mathrm{~km}^{2}$, with 88 pickup points containing 200 waste bins. This vehicle used a global positioning system (GPS) tracking device, which made it easier and more accurate to identify the route traveled. The ArcGIS geodatabase developed in this study consisted of: raster photo images of the city, street network data, waste bin locations, waste bin capacity, current collection routes driven, service time to collect bins, truck type, and capacity. The original collection route length was $30.9 \mathrm{~km}$, while the new method produced a waste collection route of $22.2 \mathrm{~km}$ in length, for a $28.1 \%$ decrease in overall kilometers traveled. This created a potential savings of $2,710 \mathrm{~km}$ driven per year, based on the vehicle's schedule of six collections per week. The decrease in $\mathrm{CO}^{2}$ emissions was also calculated, based on the reduction in kilometers driven the city would produce 40 less tons of $\mathrm{CO}^{2}$ emissions each year.

Research shows fuel consumption optimization can return substantial savings to a municipal solid waste management program. A 2007 case study by Tavares, et al used GIS technology to evaluate solid waste collection fuel savings through route optimization. This study differed from others since it factors in 3D topographic relief. The terrain for each road segment was used as a cost factor based on the slope value. Most route optimization studies focused analysis on calculating the shortest distance or shortest drive times for waste collection and transport. The study focused on the Island of Santo Antao, the westernmost island of Cape Verde, which lies off the western coast of Africa. Santo Antao covers $779 \mathrm{~km}^{2}$ with a population of 49,000 people. The island is one of 10 islands in Cape Verde and was chosen for the study because of the rugged topographic relief, with its highest point reaching $1,979 \mathrm{~m}$ above sea level. The first phase involved combining 3D terrain data with the 2D road network. This allowed calculation for inclinations of each road segment, which was integral in fuel consumption analysis for uphill and downhill directions. Phase 2 involved calculation of actual fuel consumption during waste collection and transport. The method used factored in road gradient, truckload, diverse driving conditions, and vehicle parameters. The software tool COPERT was used to calculate air pollutants and greenhouse gas emissions discharged from the transport trucks. Phase 3 of the study focused on optimizing the route sanitation trucks traveled to each collection point on the island. Esri's ArcGIS Network Analyst extension was used to calculate the least cost path for vehicles to traverse. When terrain was factored as a route cost impedance there was an increase of $34 \%$ kilometers traveled but also a $52 \%$ drop in total fuel consumption. This was because of the optimized route avoiding steep climbs over high topographic relief roads. The route would follow the flat lower elevation roads along the coast, which increased overall mileage but substantially 
reduced vehicle fuel consumption. This study shows the importance of factoring in terrain relief as impedance, when optimizing for a waste collection route in a high topographic relief area.

Overall, research shows implementing a route solving solution using advanced software can return substantial cost savings. This is accomplished by reducing fuel costs, decreasing mileage driven, or a drop in overall travel time.

\subsection{Summary}

The municipal waste management community clearly identified their need for more capable means to optimize solid waste collection and transport. With the rising fuel costs, labor, vehicle maintenance, and increased volume of solid waste from population and consumption growth, this continues to challenge planners to find innovative solutions to reduce overall costs. Planners have focused specifically on the waste collection and transport phase, which tends to be the most costly stage in a municipal waste management program. Optimizing the collection route with advanced routing software is a common approach to reducing travel costs. There are many routing programs available for conducting route analysis and optimization. This study focused mainly on the use of Esri products, Network Analyst extension to ArcGIS, and Vehicle Routing Problem solver, since this is the preferred software by the client. The Vehicle Routing Problem solver in Network Analyst has many advanced features and allows users to customize different parameters already built on a GIS platform. This provides an expedient solution for many different routing scenarios. 



\section{Chapter 3 - Systems Analysis and Design}

This chapter identifies the general project design and analysis used to solve the client's problem. The requirements analysis section identifies both functional and non-functional requirements needed to conduct the project analysis. The system design section describes the software, hardware, and major design components of the project. The chapter concludes with a complete description of the project plan which explains the project's main tasks.

\subsection{Problem Statement}

The city of Redlands, California wanted to use GIS technology to reduce costs and increase efficiency of collecting and transporting solid waste from residential customers' homes. A large part of a city sanitation budget goes towards fuel, maintenance, and labor for their fleet of sanitation collection vehicles. The City of Redlands wanted to use GIS technology to help reduce their overall costs. The city was not sure how to design better travel routes to enhance efficiency, as it is a complicated problem involving many influencing factors. Some of those factors are location of waste bins, collection details, operational hours, driving habits, type of vehicle, travel impedances, and integrity of road network being traversed. The city wanted to know the best approach and configurations using a GIS to help optimize sanitation collection routing.

\subsection{Requirements Analysis}

The requirements analysis is concerned with the functions that are necessary to complete the goals and deliverables for the project. This includes both functional and nonfunctional requirements. The functional requirements are based on elements the project needed to be successful. The non-functional requirements concentrate on the technical, operational, and transitional requirements that reinforced the main components.

\subsubsection{Functional Requirements}

The most critical functional requirement of the system was to calculate optimal routes for solid waste collection with various settings, such as vehicle capacity, service time, and break time. The outputs should show travel distance and drive time and then comparisons among different resulting routes could be conducted for the different scenarios. To be able to calculate optimal routes, the system was also required to store service order locations and depot locations. In this project, the service order locations for the study area were identified based on the residential rooftops, and the depot locations were based on the municipal yard start point and landfill unloading point. A road network dataset representing all the streets within Redlands was also needed to be compiled in order to properly perform route optimization calculations through the ArcGIS Network Analyst extension. The road edges and junctions had to have coincident geometry for the route optimization calculations to perform properly. Finally, the existing collection route 
needed to be compiled for comparing current and new routes. The functional requirements are listed in Table 3-1.

Table 3-1. System Design Functional Requirements

\begin{tabular}{|l|l|l|}
\hline \multicolumn{1}{|c|}{$\begin{array}{c}\text { System } \\
\text { Requirement }\end{array}$} & \multicolumn{1}{c|}{ Requirement } & \multicolumn{1}{c|}{ Description } \\
\hline $\begin{array}{l}\text { Residential Customer } \\
\text { Locations }\end{array}$ & Functional & $\begin{array}{l}\text { Rooftop point locations of } \\
\text { current residential customers } \\
\text { within the collection district }\end{array}$ \\
\hline Road Network Dataset & Functional & $\begin{array}{l}\text { A complete network dataset } \\
\text { of all the roads within the } \\
\text { city limits }\end{array}$ \\
\hline Route Optimization & Functional & $\begin{array}{l}\text { Calculation of newly } \\
\text { optimized collection route } \\
\text { for residential waste }\end{array}$ \\
\hline Existing collection route & Functional & $\begin{array}{l}\text { Location of existing route } \\
\text { traveled to service customers } \\
\text { in collection study area }\end{array}$ \\
\hline
\end{tabular}

\subsubsection{Non-Functional Requirements}

The non-functional requirements are described in Table 3-2. Non-functional requirements represent the technical, operational, and transitional aspects of the project that were essential in performing the analysis and final deliverables. A Windows 7 operating system was the preferred technical requirement, although a Windows XP operating system would have been sufficient. The hardware specifications for this project included a desktop or laptop computer with a minimum processor speed of $2.2 \mathrm{GHz}$ with $4 \mathrm{~GB}$ of RAM. The project performs complex calculations to determine optimized routes; therefore any increase in the size of RAM would substantially improve overall computer performance and processing speeds. Thorough knowledge of ArcGIS software version 10.1 was an operational requirement, since this project required complex construction, editing, and analysis in a GIS environment. Knowledge of the latest software version, 10.1, was helpful since some of the current features were used throughout the project; however, a thorough knowledge of previous ArcGIS software versions would be acceptable. A comprehensive understanding of ArcGIS Network Analyst 10.1 extension was needed to properly structure components and accurately design, construct, and perform analysis using problem solvers. The non-function transitional data used in project construction were in file geodatabase format, compatible with Esri's ArcGIS software version 10.1. Tables and guidance documents were constructed in Microsoft Excel and Word 2010 formats and were for non-function transitional purpose. 
Table 3-2. System Design Non-Functional Requirements

\begin{tabular}{|l|l|l|}
\hline \multicolumn{1}{|c|}{$\begin{array}{c}\text { System } \\
\text { Requirement }\end{array}$} & \multicolumn{1}{|c|}{ Requirement } & \multicolumn{1}{c|}{ Description } \\
\hline $\begin{array}{l}\text { Esri ArcGIS Desktop } \\
\text { Software Version 10.1 }\end{array}$ & Non-functional: Technical & $\begin{array}{l}\text { ArcGIS program with } \\
\text { Network Analyst extension } \\
\text { enabled and functioning } \\
\text { problem solvers }\end{array}$ \\
\hline Operating System & Non-functional: Technical & Windows 7 or Windows XP \\
\hline System Hardware & Non-functional: Technical & $\begin{array}{l}\text { Desktop or laptop, Intel } \\
\text { processor recommended } \\
\text { 2.2GHz, 2GB RAM }\end{array}$ \\
\hline Experience level & $\begin{array}{l}\text { Non-functional: } \\
\text { Operational }\end{array}$ & $\begin{array}{l}\text { Thorough knowledge of } \\
\text { ArcGIS software with } \\
\text { fundamental knowledge of } \\
\text { Network Analyst extension }\end{array}$ \\
\hline Data format & $\begin{array}{l}\text { Non-functional: } \\
\text { Transitional }\end{array}$ & $\begin{array}{l}\text { All final data must be } \\
\text { configured for ArcGIS }\end{array}$ \\
\hline Documentation & $\begin{array}{l}\text { Non-functional: } \\
\text { Transitional }\end{array}$ & $\begin{array}{l}\text { The methodology should be } \\
\text { documented for further } \\
\text { analysis usage }\end{array}$ \\
\hline
\end{tabular}




\subsection{System Design}

The system design for this study was based on a requirements analysis and designed to accommodate the project's principal components. Figure 3-1 displays the project's major components and how they integrated into the workflow.

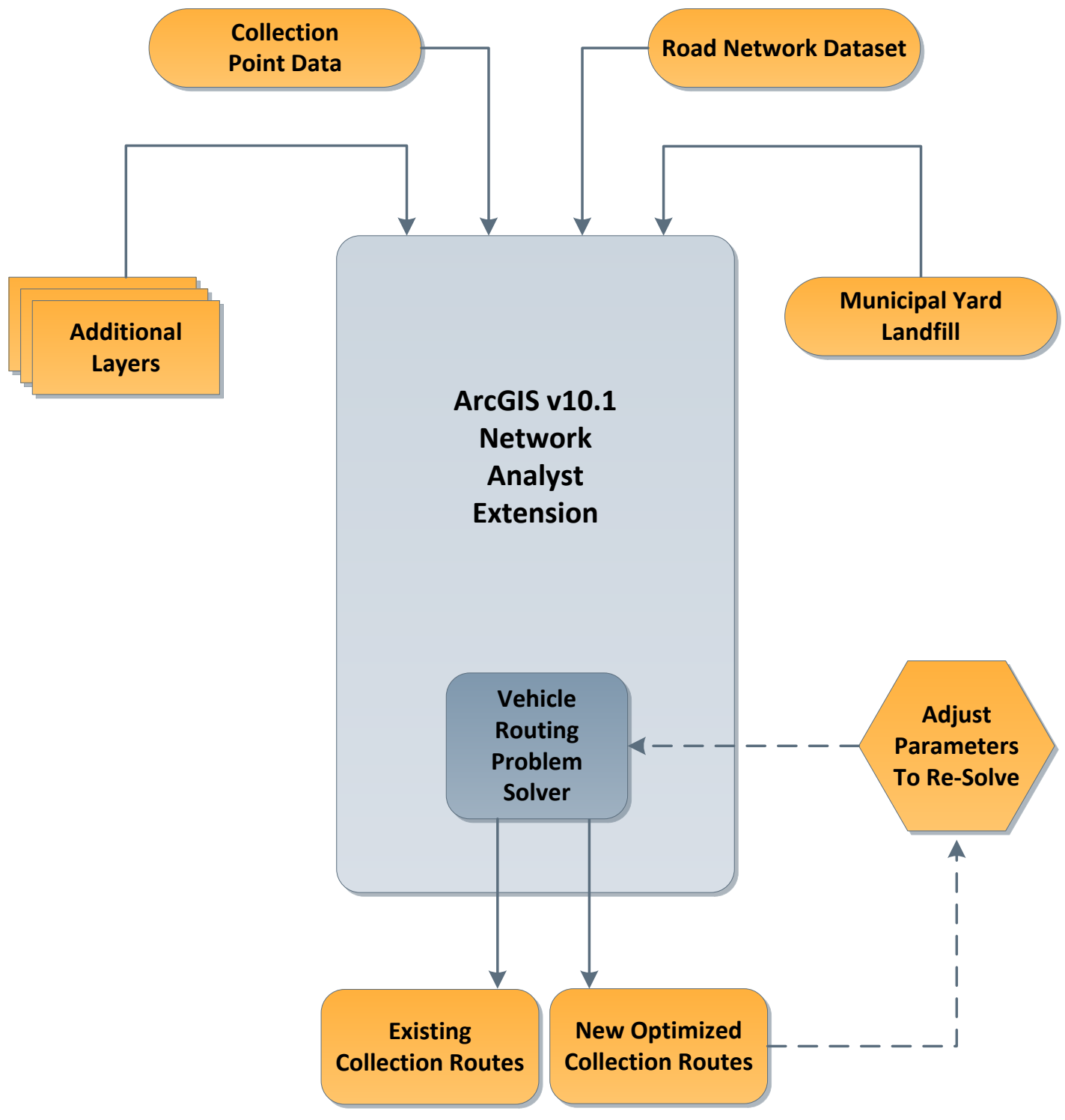

Figure 3-1: Project System Design 
To calculate optimal routes for solid waste collection, there were several inputs to the ArcGIS Network Analyst VRP solver. Both collection points represented by the rooftops and depot locations including municipal yard start point and landfill unload point were required. A network dataset of Redlands roads was constructed to assure all street segments are connected at junctions. Additional GIS layers were necessary to be incorporated into the VRP solver for accurate representation of potential routes traveled. For example, stop signs and traffic lights were included to better estimate impedance encountered by the truck driver. A raster DTM file was imported into ArcGIS and included the elevation and topographic relief information for the study area. These data were used to perform test calculations of slope for each road segment within the city. Once the input layers were determined, different parameters needed to be configured on the VRP solver, including: time windows, service times, break times, vehicle capacity, cost barriers, and restrictions. With all the inputs and parameters set up, the VRP solver can construct a new optimized route with optimal sequencing. The route was evaluated for accuracy and route parameters were then adjusted accordingly. Multiple route solver iterations were run until an optimal collection route was identified.

\subsection{Project Plan}

The project plan was developed to help keep the project on track. Milestones and a timeline were created to keep track of progress throughout the project life cycle. The project plan was created using the waterfall project management approach. Waterfall is a sequenced design process in which each project phase is dependent on previously completed steps. This approach was chosen for its simplicity, efficiency, and reliability which turned out favorably because of the project time restraints. There were five key phases to the project: planning, research, data review, analysis, and finalization. The following sections explain each phase in more detail.

\subsubsection{Project Planning}

The project planning phase included discussions with the client to gain a thorough understanding of the problem to solve. How project results could be used for future enhancement on remaining collection routes was discussed. The planning also involved discussions with Quality of Life personnel, since the QOL would be directly impacted by any future route changes made. Requirements analysis were constructed and reviewed with the client to assure needs were understood and could be achieved. The project scope was defined and a reasonable timeline was established.

\subsubsection{Research}

Extensive and thorough research was conducted for the project, including investigation of similar case studies which used GIS or other technologies to generate optimized routes for solid waste collection. It was difficult to find literature for the recent Vehicle Routing Problem technology specific to this use, since it is a relatively new tool, but Esri had substantial documentation available on the capabilities of the software extension. There were several studies done on the use of Esri Network Analyst extension as a planning tool in a solid waste management program. The research also showed a growing number 
of route optimization programs being introduced into the software market, along with several companies customizing routing software to meet a client's specific needs. A common element between many research documents was that implementing a successful route optimization plan into an SWMP has potential to identify large cost savings. These savings can result from: cuts in overall miles driven, decreases in travel time, increasing collection points, reducing wear and tear on vehicles, and a cut in overtime hours.

\subsubsection{Data Collection and Review}

The data collection and review phase involved identifying the datasets needed to successfully complete the project. All datasets obtained were reviewed thoroughly for quality and consistency. The residential collection point data needed several edits performed. This involved removing duplicate and outlier points beyond the study area. A visual inspection was done to ensure all points aligned on the building rooftop within each property parcel. The dataset for all the city roads required a considerable number of edits. The process involved comparing the project road data obtained with a completed set, street base maps, and current aerial imagery to identify any missing road segments. The discovered missing road segments were manually digitized back in using the edit functions within ArcGIS. Many routing optimization projects use road data supplied by NAVTEQ NAVSTREETS, TomTom MultiNet, or Esri StreetMap. This project required the eRoadInfo data since they included important attribute data corresponding to each road segment. These data were integral for the analysis since multiple attribute fields were used during the route optimization calculations. Additional data were obtained during multiple client meetings. These data related to vehicle and driver specifics such as: truck model, driver habits, capacity, fuel type, driver work hours, current route driven, and start and finish locations.

\subsubsection{Project Implementation and Analysis}

The project implementation phase consisted of updating and importing data and layers into ArcGIS. Route solver parameters were adjusted accordingly to meet client requested criteria. A network dataset of the roads was configured and created. The collection points in the study were divided into smaller groups for more efficient processing during analysis. The Vehicle Routing Problem solver was started and configured with the start and finish depot points on the route. Collection point order locations were imported into the VRP solver and extra settings were configured on the layers. The Network Analyst solve tool was executed to model routes based on the current settings and criteria. The routes were assessed for quality, consistency, and practicality, and parameters were adjusted. More solve routines were run until desired output routes were achieved.

\subsubsection{Project Finalization and Delivery}

A guidance document on the GIS methods used to model optimized routes was generated. The client will reference this document to perform route optimizations on remaining collection districts in Redlands. An ArcGIS 10.1 compatible file geodatabase was delivered to the client. This included the newly optimized route and any associated datasets used for the analysis. Extra data, tables, and documents relevant to the project 
were included as a digital deliverable transferred to a flash drive. A hard copy printout of the newly optimized route and turn-by-turn directions were delivered to the client and the project was finalized.

\subsection{Summary}

This chapter discussed the system design and project plan, and provided a brief review of the problem. The system design identified and included both functional and nonfunctional requirements which helped the project achieve the needed results. The project plan was presented, outlining the five phases of the project (Planning, Research, Data Collection, Implementation, and Finalization). 


\section{Chapter 4 - Database Design}

This chapter discusses the conceptual and logical database models that were designed for the project. For GIS projects to be successful, a well-designed geodatabase should be created. A geodatabase is essential to efficiently model geographic objects, relationships, and their attributes. A conceptual data model is designed to determine the entities, attributes, and relationships among the entities. The logical data model is the physical construction of the geodatabase. The remaining sections discuss data sources, collection methods, and scrubbing and loading.

\subsection{Conceptual Data Model}

The conceptual data model defines the relationships between the different entities. The entities considered in this project include: waste bins, street network, traffic lights, stop signs, truck and driver information, existing route information, start, finish, and unload locations. Figure 4-1 illustrates the conceptual model design.

The project relies on analysis over an assembled network of connected edges representing all roads within the City of Redlands. The sanitation truck departs from the municipal yard travelling along the roads, collecting trash from residential waste bins placed along curbs in front of each home. The direct effort to unload each waste bin is calculated as the service time. The truck encounters various impedances when travelling along the network including travel time, distance, stop signs, and traffic lights. Once the truck reaches full capacity it returns to the landfill to unload the contents and then continues collection along the route until all bins are emptied. 


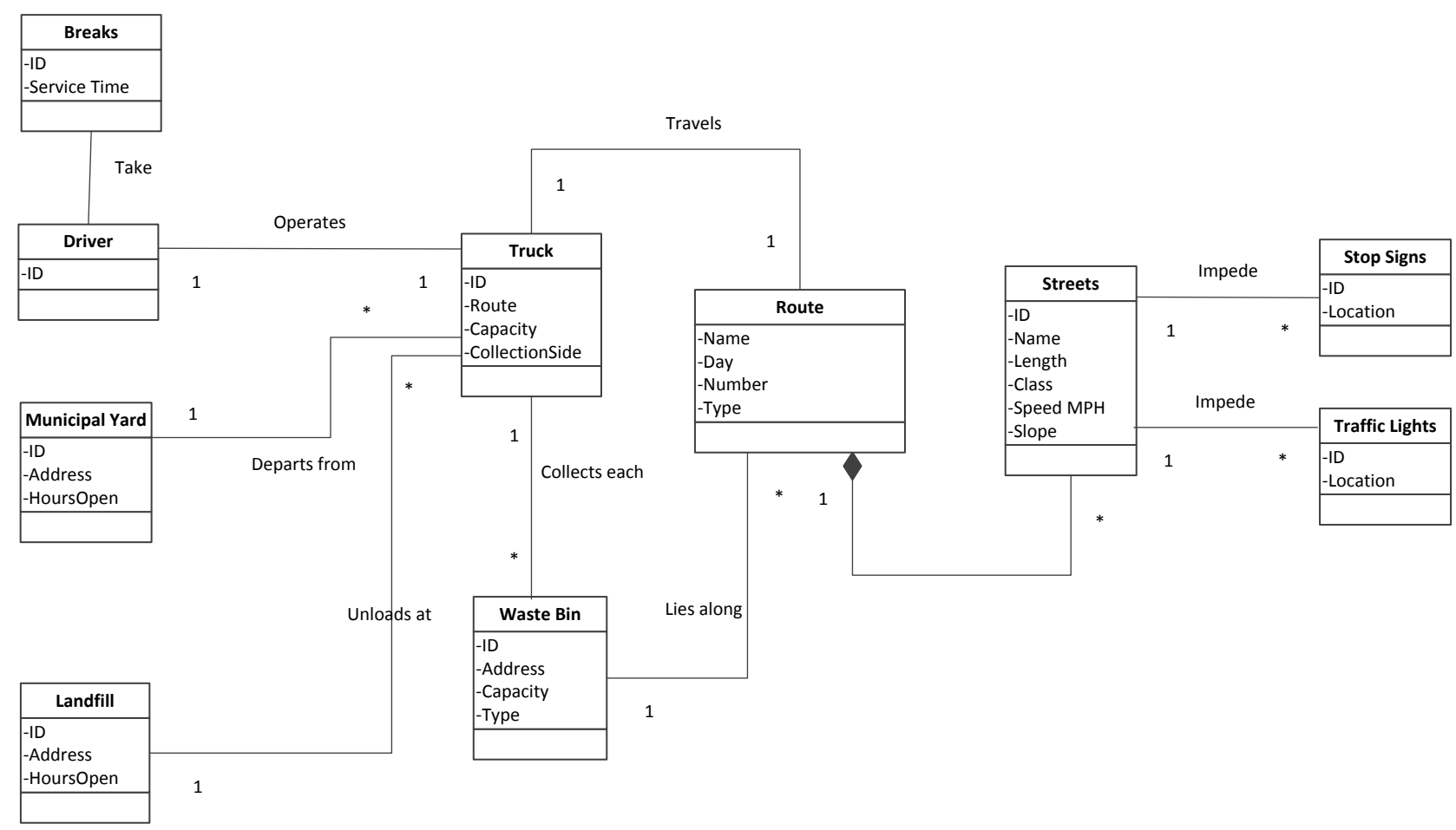

Figure 4-1: Conceptual Data Design Model

\subsection{Logical Data Model}

The logical data model takes the conceptual model and references it to construct a physical database. This project does not have a complex or large database. It uses an Esri file geodatabase format, which is a reliable database format with no file size restrictions, and can support multiple users to access when needed. The client maintains a GIS department with multiple users, who need to access the data to continue route optimization analysis for the remaining 22 collection districts in Redlands. Figure 4-2 illustrates the logical model design. 


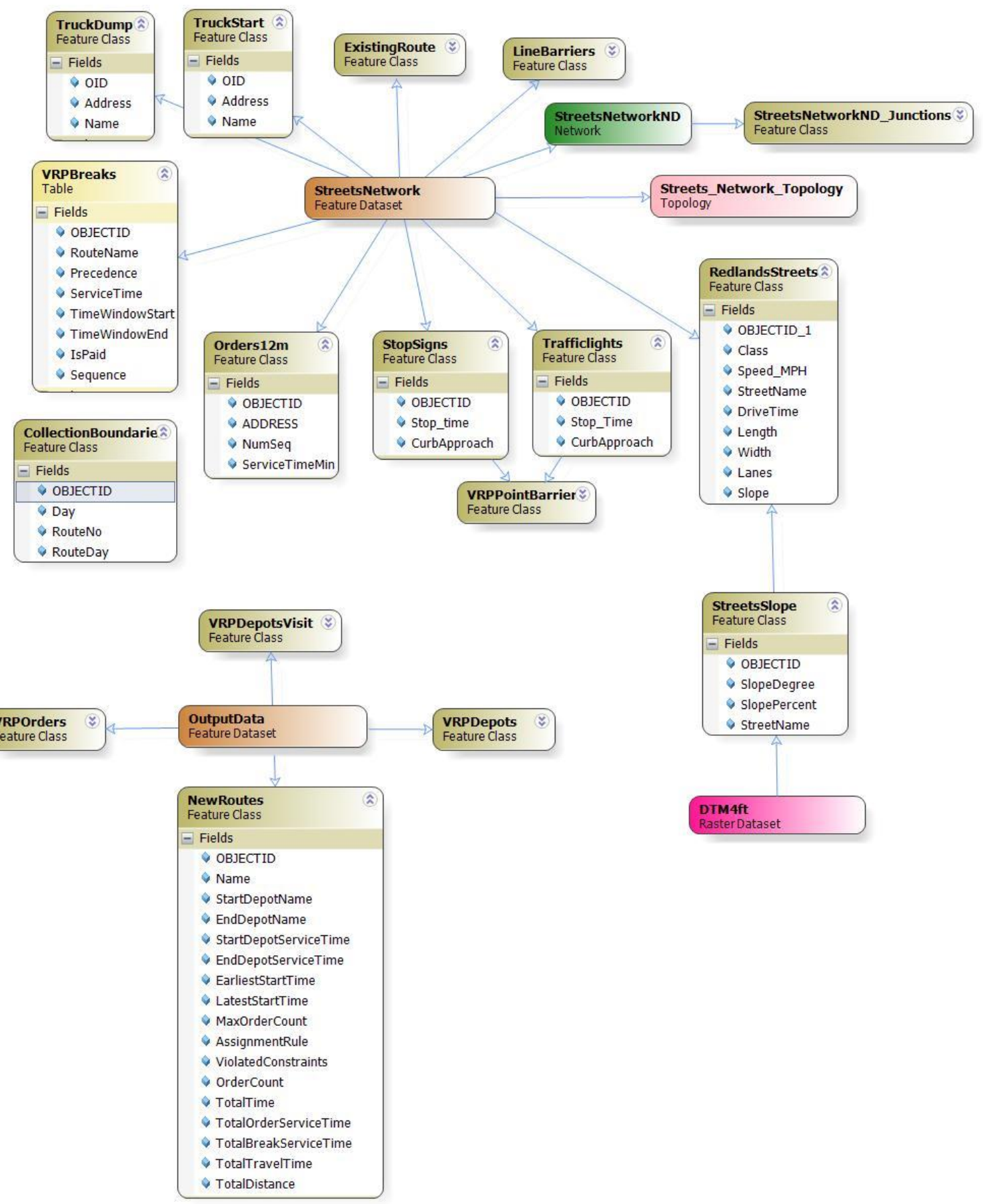

\section{Figure 4-2: Logical Design Model}

A subcategory feature dataset was created in the project geodatabase to contain all spatial features used to build the street network dataset. This was required to ensure network dataset integrity and allowed the use of topology rules. The collection points 
were also maintained inside the feature dataset. The collection point feature class was derived from a database of residential customers' rooftop locations and was included in the VRP solver as orders. This point feature class was named Orders $12 \mathrm{~m}$. The street line feature class data were the source data to build the network dataset. Attributes of the street object include: name, speed limit, slope data, drive time equations, predefined route number, and bin sequencing values. These attributes are significant to analysis since the VRP solver recognizes some as cost impedances during processing. Once the network dataset is built a junction point feature class is created. These points connect edges and facilitate navigation between all edges along the network. The junction point data are stored in the feature dataset. DTM raster images were used to develop the slope data for each corresponding street segment. The image is in the main geodatabase folder to simplify any geoprocessing steps. The existing route is a line feature class that represents the current route driven to collect residential waste. These data are kept with the feature dataset and used to compare against the newly optimized routes. The start and unload point features were defined as order and depot points when introduced to the VRP. Once the VRP solver was run, the orders and depots were stored in the map document as layers. The data were exported as point feature classes and stored in the file geodatabase. The Vehicle Routing Problem solver produced multiple line feature classes representing the existing output and new optimized routes. The existing route output includes additional attributes identifying travel time and distance values. These data are stored in the feature dataset.

\subsection{Data Sources}

All project data were collected from one of three sources. The client for this project was the City of Redlands. The city has its own functioning GIS department which supplied the collection districts, parcel data, stop signs, traffic lights and raster DTM data in a GIS compatible format. The Redlands Quality of Life Department, which oversees city sanitation collection, supplied data specific to the sanitation collection process. The data included operational hours, vehicle capacity, fuel type, driver information, existing collection routes, city landfill locations, and current collection patterns. The QOL supplied the residential collection locations in table format. The customer addresses were based on rooftop data locations and saved as point feature classes. The project required road data features for the entire City of Redlands. The original street data used for the project were obtained from eRoadInfo. In 2010 the city contracted eRoadInfo, to perform a pavement condition index report for all the streets in Redlands. These data were supplied by eRoadInfo, a pavement asset management company who performs mobile data collection of roadside assets and pavement conditions. These results are delivered in report form along with completed GIS datasets. The report provided detailed analysis of each road segment with matching attribute information such as: road width, lanes, pavement condition, speed, shoulder width, classification, name, and surface type. 


\subsection{Data Collection Methods}

The data collection method for this project was straightforward. The digital data were obtained through multiple meetings with the client and transferred to an external USB hard drive. The information regarding waste collection practice in the city was obtained via field observations and correspondence with sanitation personnel. The information included: vehicle type, operational hours, service time, general department logistics, driving habits, and overall functionality of the solid waste collection process. Further, the existing collection routes were constructed from discussions with drivers then sketching collection patterns onto the city maps. This information represented the turn-by-turn route driven to collect waste bins in the study area. The information was also confirmed through visual inspections of the collection route. This provided a better understanding of the collection process such as service time to collect bins, approximate time to cover a distance, and overall driver behavior. The existing routes sketched on the paper maps were then digitized into vector lines and stored in the file geodatabase. Several field observation sessions were conducted to evaluate the collection process and research driver techniques and patterns.

\subsection{Data Scrubbing and Loading}

The original dataset for the streets required a substantial amount of editing and updating. For Network Analyst to accurately solve a routing problem, the street data used by the solver must have geometric connectivity between all lines. During route analysis, this allows the solver to access every road segment on the network. If street line segments are not connected by end points, the solver identifies the segment as a dead end and a continuous path cannot be calculated along that segment. To resolve this issue the integrate tool in ArcGIS desktop was used. This tool maintains integrity between feature boundaries by assigning an allowable tolerance. If features fall within the specified tolerance they are considered coincident and a vertex is added to each edge, creating a connection. There were several network edges that lay outside of the five foot buffer area. Once identified, these segments were joined during edit sessions by manually connecting edges. The street data were also missing many individual road segments, which was discovered during the quality check phase by including a street base map layer and aerial imagery underneath the project data and visually searching for missing segments. Although this was a very tedious process, it was imperative to have all major road segments within the city included to have better route optimization results. The missing road segments were digitized based on tracing the sections from the ArcGIS streets base map layer. In the edit sessions, the attributes for the digitized streets were manually updated accordingly.

Collection point data representing residential waste bins had many duplicate fields and required review and editing to remove identical records. This dataset also had several outlier points that were beyond the collection district study area. These points were manually removed from the dataset.

Stop sign and traffic light point feature classes were edited using Topology Rule Point Must be Covered by Line; this assured these impedance points were coincident with a corresponding road segment along the network. 


\subsection{Summary}

This chapter discussed the conceptual and logical models of the project database. The conceptual model section discussed project data classes and attributes, as well as their relationship to each other within the database. The logical model section described how the physical database was constructed based on the conceptual model design. The data source section discussed where and how the data needed for the project were obtained. The final section of data scrubbing and loading, described the integrity of obtained project data and the approaches taken to assure data meets the quality standards needed to properly perform analysis. 


\section{Chapter 5 - Implementation}

This chapter presents the method used to create optimized collection routes, as well as the implementation process. Creating an optimized route in ArcGIS required data preparation, configuring network datasets, building network datasets, and performing route optimization analysis (Figure 5-1). The Vehicle Routing Problem solver provided by the ArcGIS Network Analyst was used to produce new collection routes. For this project, orders, depots, routes, breaks, and point barriers were considered to generate new collection routes and multiple settings for these input parameters were experimented.

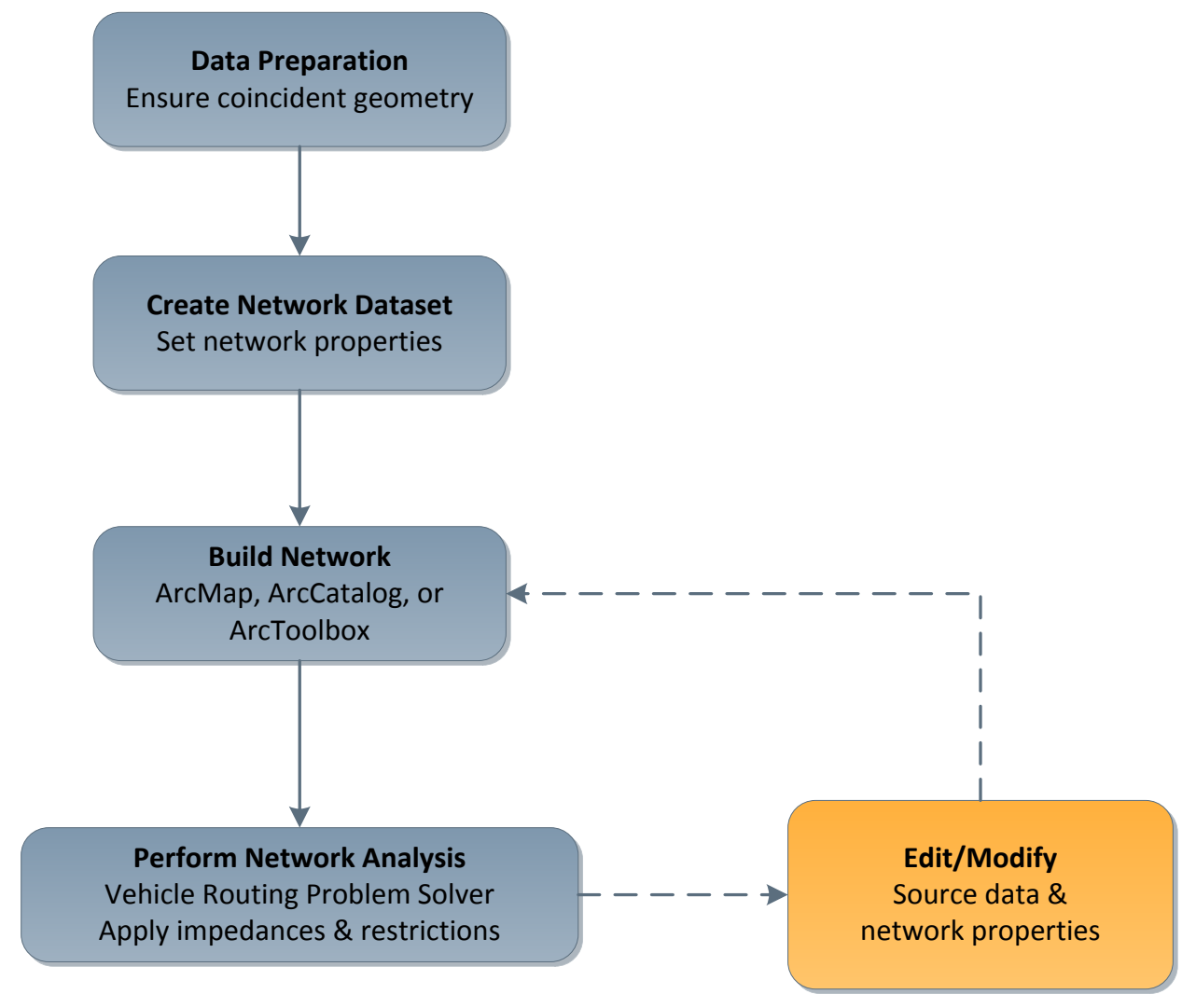

Figure 5-1: Network Analyst Vehicle Routing Problem Solver Workflow

\subsection{Road Slope Analysis Experiment}

Given that solid waste collection vehicles normally have a large load, fuel consumption may be greatly affected by the slope of the streets. Thus, a road slope analysis was first attempted at the beginning of the project. The intent was to have slope values recognized as cost impedances on the network dataset. The 4 foot digital terrain model (DTM) was used during geoprocessing to reference the elevation values stored in 
each grid cell. The workflow was accomplished in ArcGIS 10.1 and is described throughout this section. Figure 5-2 illustrates the slope analysis experiment workflow. In order to calculate slope along the street centerline, a buffer around the centerline was used as a mask to extract grid cell values from the DTM. Initially, the buffer was generated based on the road width attribute field, with values ranging from 15-100 ft. The Spatial Analyst Slope tool was used to calculate both degree and percentage slope values. After reviewing the slope result on the map it was decided to use a narrower width for the buffer. This was becausesome steep slopes were found wherever roads were adjacent to sharp vertical embankments. The concern was, when averaging slope for road segments, these values would skew results. A six-foot buffer was then created based on each road's centerline. This gave a more accurate representation of slope values just for paved sections of each road segment.

$<$ Figure 5-2 here >

The Raster to Polygon tool was used to convert the new slope values to a vector format for integration into the street dataset. Slope values were reclassified into three classes and the intersect tool was used to join values to the road feature class attribute table. Another approach to obtain elevation values for each road segment was to use the new Stack Profile tool from the ArcGIS 10.1 3D Analyst extension, which defines profile of line features over terrain surfaces. The tool provided the desired data results by extracting elevation values from the DTM based on intersecting road line segments; however, it had limitations when trying to perform the process on multiple road segments at one time. The intent was to use the tool in the ArcGIS ModelBuilder application to extract elevation values for all individual road segments. These elevation values could be used for calculating slope for each segment, as well as for confirming the earlier slope results. Unfortunately, this tool was unsuccessful in calculating slope for multiple line features at one time.

Upon reviewing the slope data and conducting further research on calibrating a transportation network with terrain information for routing, the decision was made to exclude slope data from the analysis. Including 3D terrain information into GIS route optimization analysis involves several complex steps. First, a three-dimensional road network needs to be constructed. This involves getting $\mathrm{X}, \mathrm{Y}$, and $\mathrm{Z}$ values for each road segment and becomes more complex when factoring in bridges, tunnels, and overpasses along the transportation network. Then the difficulty in traversing a street segment due to terrain variability needs to be categorized. This is done to identify if travel along that network segment is uphill or downhill. This involves calibrating network edges with directional impedance attributes for the distance travelling uphill. Just having the slope value for each road segment is not enough; since slopes are directional, a bi-directional attribute would need to be created for each segment as well (Sandhu, 2011). The time frame for this project was insufficient to construct the necessary 3D network to implement in the VRP solver analysis, but this could be a very valuable future work to be completed. 


\subsection{Road Network Dataset for Route Analysis}

For Network Analyst to function properly it requires a network dataset of connected features which provide the platform for each Network Analyst solver to perform calculations and analyses. Network datasets are created from feature classes in a feature dataset of a geodatabase. This project used road feature classes created by a pavement asset management company eRoadInfo. Other road feature data were available from NAVTEQ NAVSTREETS, TomTom MultiNet, and Esri StreetMap. The eRoadInfo data were chosen for their updated attribute information specific to the City of Redlands. The company produces detailed reports on integrity of roads; included in the eRoadInfo report are GIS compatible files. These files are generated from GPS coordinate data and contain multiple useful attribute fields, such as road width, lanes, pavement condition, speed limit, classification, name, and surface type. The attribute fields are linked to individual road segments and used as different parameters in the route optimization analyses. Upon reviewing the data it was discovered that many road segments were missing. This was discovered during the quality control check by overlaying road data with current aerial imagery and street map data. The tedious process of digitizing missing road segments was conducted during multiple edit sessions. Attributes for the new road segments were updated accordingly. The following road attributes were updated: name, class, length, width, lanes, speed, and drive times. A feature dataset was created to store files associated with the network dataset and stored in the project file geodatabase. The coordinate system for the project was NAD 1983 State Plane California V FIPS 0405 Feet, with a Lambert Conformal Conic projection. The client will incorporate project data into its existing GIS which currently uses this coordinate 
system. A topology rule was created for stop signs, traffic light points, and road line feature classes. The point must be covered by line topology rule was defined and stored in the feature dataset. This rule assured all stop sign and traffic light point features were connected to road line features along the network. These points are recognized as travel impedances when the truck travels across the network.

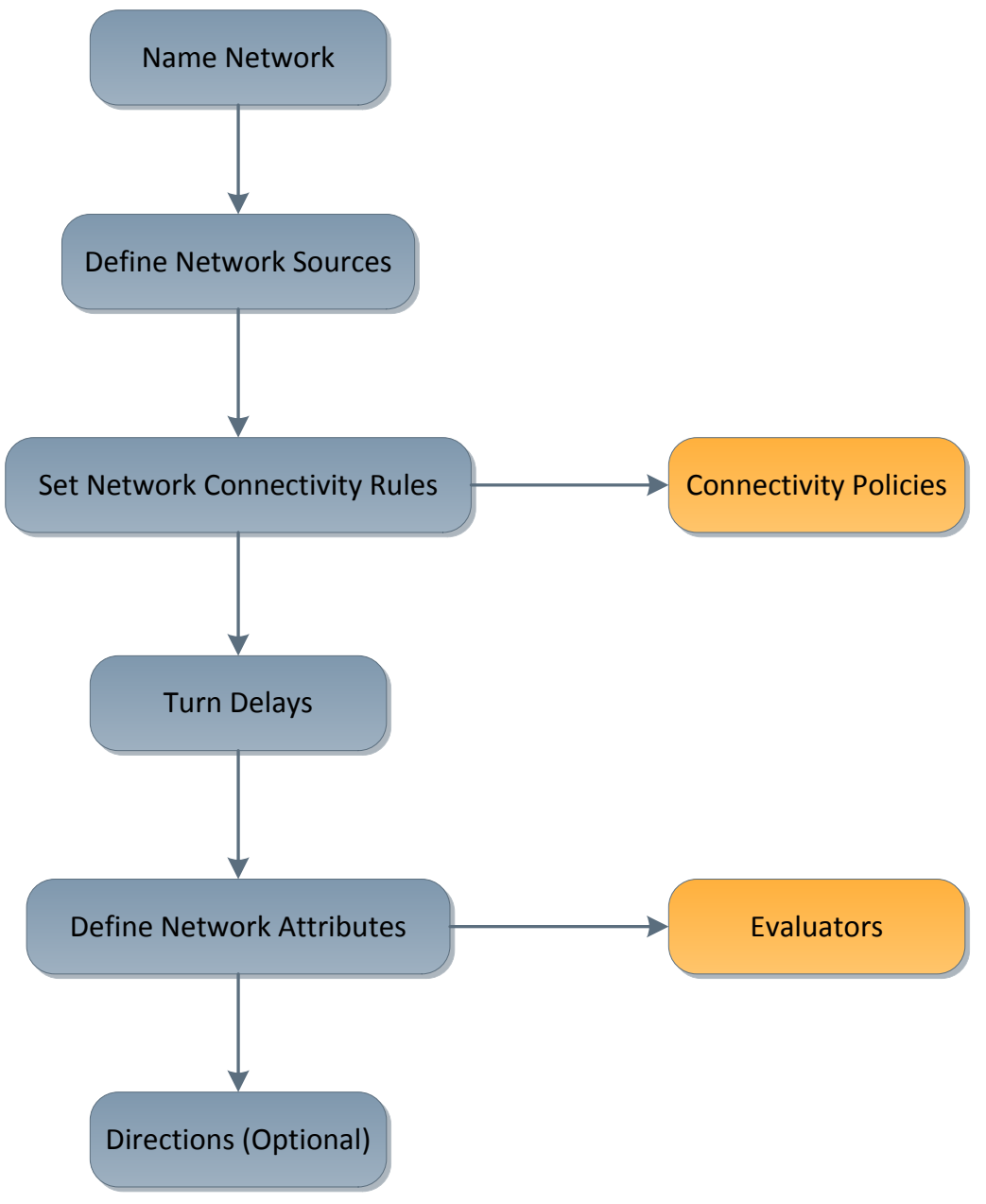

Figure 5-3: Network Dataset Construction Diagram

Figure 5-3 illustrates the network dataset construction process. When building a network dataset it is important that features have coincident geometry. The Integrate tool was used to assure all road line features on the network were connected. When all road segments are connected this guarantees their inclusion during network calculations. The 
updated road feature class was used as the network source. The network connectivity policy was set to use any vertex. Network connectivity only exists at points of coincidence between line and point features. An additional connectivity policy to consider is the elevation model. When constructing a network this allows the dataset to represent multiple levels for line features along the network. This model is used to identify bridges, tunnels, and overpasses within a network. The feature was not used since it was not applicable for the project study area.

The attributes of a network dataset define the association between network elements.

All network elements have the same set of attributes with potentially different values. The attributes control navigation through the network. The four types of network attributes are: cost, restriction, hierarchy, and descriptor. Cost attributes are the values accumulated as the network element is traversed. These values are apportioned along edges (roads) of the network. Cost attributes can be either time or distance to travel a network segment. This project calculated both drive time and total distance. Drive time was configured as an expression in the driveTime20 and driveTime40 fields of the road feature class. The expressions for these two cost fields are $80 \%$ and $60 \%$ of the speed limit attribute value, multiplied by the length of that road segment. The logic is that large trucks would rarely maintain the maximum posted speed limit for entire road segments. Sanitation collection trucks travel short sections with many starts, stops, and turns, and the trucks are large, heavy and tend to take time reaching desired driving speeds. The other cost this project solved for was distance. This cost was configured to read the miles length field from each road segment. The Network Analyst Route Solver calculated the shortest route without factoring in any drive time concerns. This project used a restriction attribute to identify roads on the network that should not be included during analysis. The restriction was labeled RestrictedRoads and references the Class field of the road features. The RestrictedRoads evaluator has an expression built to select private roads from the class field, which excludes all private roads from the network calculations. The 
restriction attribute was created to exclude park and cemetery roads in the study area during analysis.

The network dataset had a global turn delay evaluator configured to apply time penalties for different turns made on the network (Figure 5-5). The network has a five second time delay assigned to any left turns made on the network. When solving a route for time, this increased value tells the solver to find a quicker network segment to traverse. The VRP solver did not avoid all left-hand turns; it just calculated these turns as extra effort to navigate. Route optimization studies have shown that minimizing left-hand turns for a large fleet of vehicles can help improve overall fuel efficiency (Spector, 2013). Research has shown in certain situations vehicles will burn more fuel waiting for traffic while trying to make left-hand turns. Minimizing left-hand turns may also lessen the likelihood of accidents by reducing the need to cross one or more lanes of oncoming traffic (Lovell, 2007).

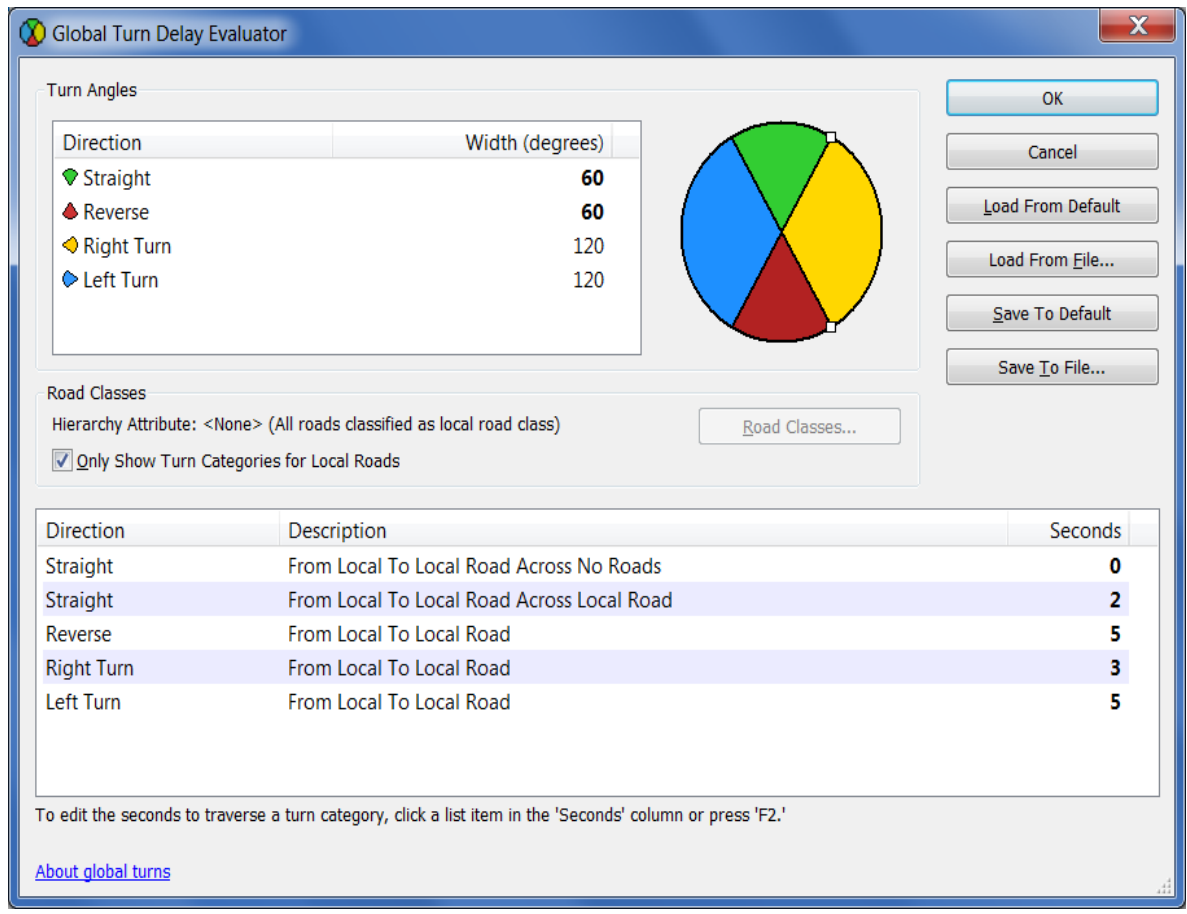

Figure -5: Global Turn Delay Evaluator

The directions feature was enabled within the network dataset to provide turn by turn instructions for the completed routes. The directions were part of the client deliverables and provide the drivers a detailed reference for traveling each route. 
The network dataset was then built and stored in the feature dataset. Point feature classes of all network junctions were created and stored with the network dataset. The junctions define connections between edges on the network and are important for defining continuity throughout the entire network. Network quality control was done by visually inspecting junction points to confirm location at each intersection. Multiple intersections were missing junctions. These edges were edited to create connections to all adjacent segments by using the integrate tool and assigning a buffer threshold to recognize, and then connect edges identified within that buffer distance. The network dataset was rebuilt and ready for use within the Network Analyst solvers.

\subsection{Vehicle Routing Problem Solver for Route Optimization}

This section discusses how the Network Analyst Vehicle Routing Problem solver was used to build new waste collection routes and explains in detail the workflow, properties, settings, and data used for route analysis and construction. Most of the settings and values described are from the initial analysis for the entire 829 points inside the study area. Additional studies were conducted with different settings and smaller groups. These results are further explained in Chapter 6. The Vehicle Routing Problem (VRP) solver is a feature in the ArcGIS Network Analyst software extension. A function of the VRP is to service orders and reduce overall operating costs for fleet vehicles. This was accomplished along a transportation network dataset of connected edges and junctions. During the analysis the VRP solver factored in different parameters, as well as cost impedances crossed while travelling along the network. The VRP has an analysis layer made up of 13 network analysis classes which is the main interface for importing classes and configuring different solver parameters. These classes represent tables and feature layers stored inside the analysis layer. The network analysis objects are used in solving the vehicle routing problem. The Vehicle Routing Problem solver workflow is illustrated below in Figure 5-5. 


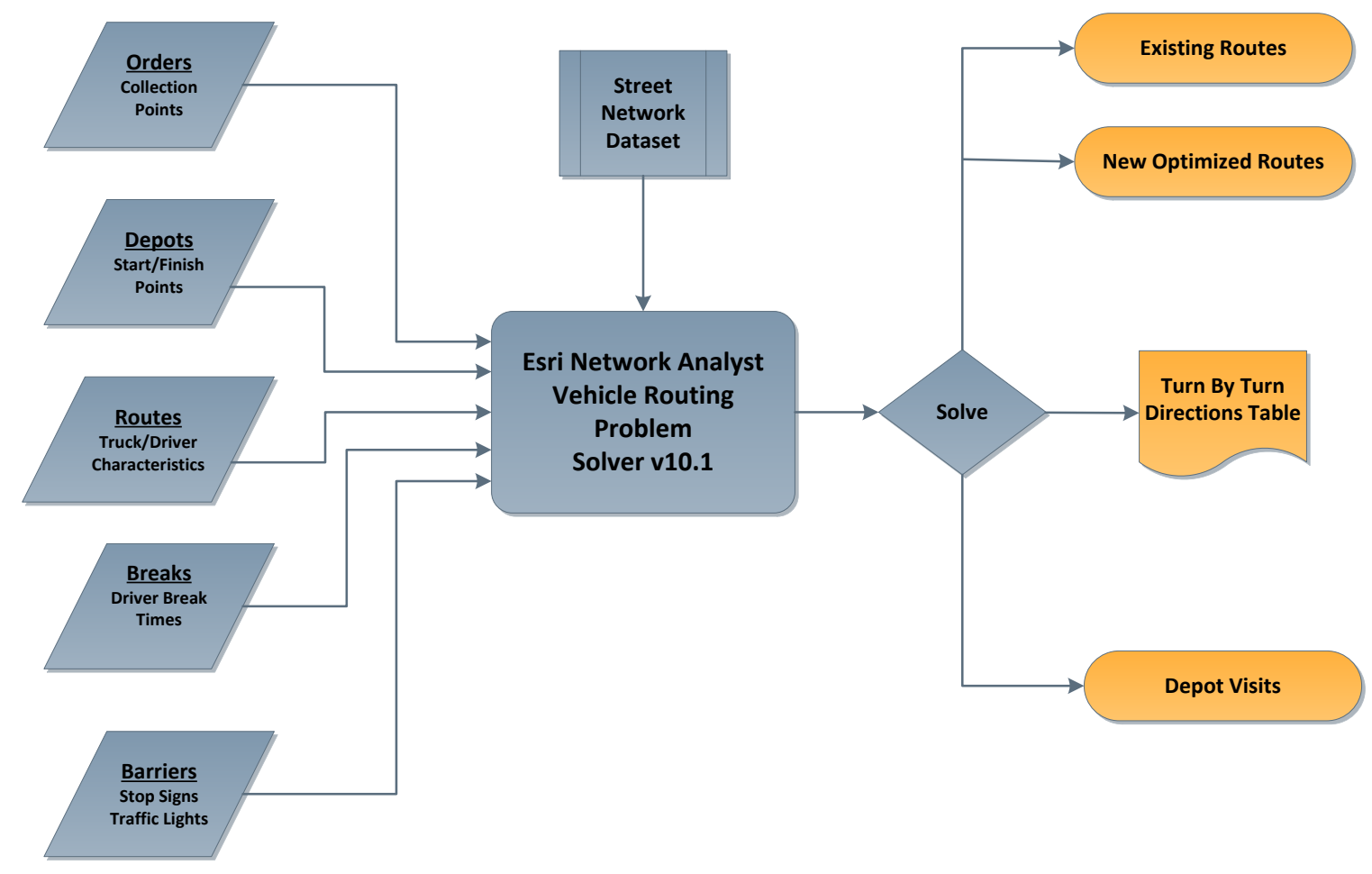

Figure -6: Vehicle Routing Problem Solver Detailed Workflow

\subsubsection{Orders}

Orders refer to pick up point locations, which are stored in a feature layer in the VRP analysis layer. The orders for this project were point features derived from a database of rooftop locations that represent the 829 residential homes with waste bins to service in the study area (Figure 5-6). 


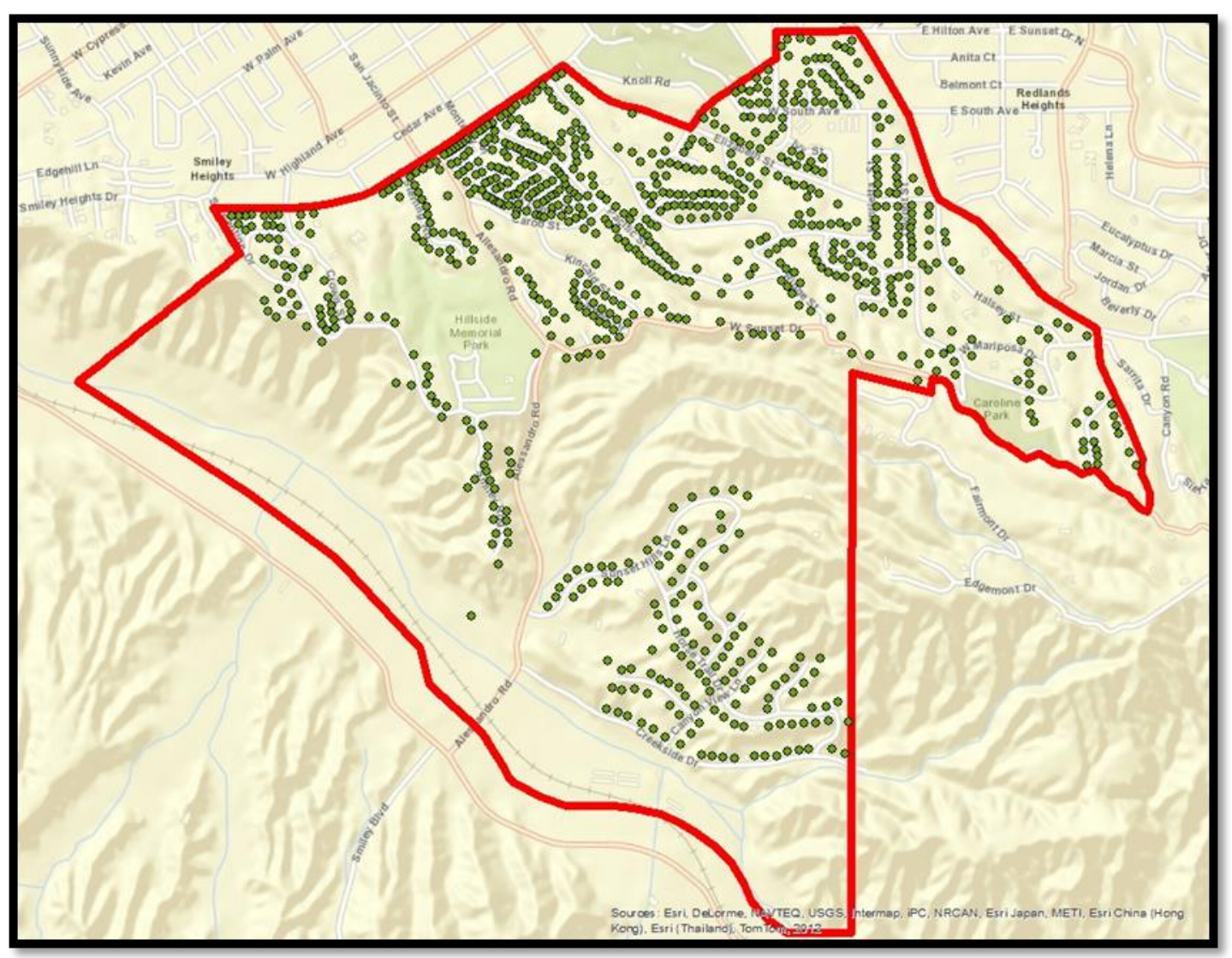

Figure 5-7: VRP Orders in Study Area

Different input fields can be defined on the order properties. The service time setting is how much time is spent at each order location on the route. This cost value is stored with each order location and was set to 10 seconds for most orders. The city provides a curbside service to retrieve waste bins from homes of the elderly and handicapped; the driver brings the bin from the home down to the curbside for collection. For these homes the order service time to unload a waste bin was set to one minute. This value was supplied by the Quality of Life Department based on the average time trucks take to load waste bins and was also verified by field observations of trucks collecting along the route. The time window start and end properties were set to 6:00 am to 2:00 pm for each order, which reflects the driver's standard workday Monday through Friday. The orders curb approach setting was configured to right side. This is based on the city sanitation trucks that are automated to collect waste bins from the vehicle's right side. Once the solver was run, the order feature was updated to include route name, sequence number, and arrival time attribute values.

Upon reviewing the rooftop point location data, it was discovered several points were snapped to network road edges that did not reflect the correct road segments where a bin would be collected. This consistently happened for homes with long driveways and smaller backyards (Figure 5-8). When a route solver is configured over a network dataset, the orders are identified and snapped to the closest network edge. This tells the solver 
exactly where the order point lies along the network and how to travel the network to access it. For this project the rooftop point data were used as the bin locations to be serviced. However, a waste bin is typically located at the end of each homeowner's driveway for collection. The majority of the rooftop points were snapped to the network edge closest to a home's driveway, but there were several snapped to network edges that did not represent where the home's driveway would intersect the network. These points were manually adjusted to snap to the correct network edge.

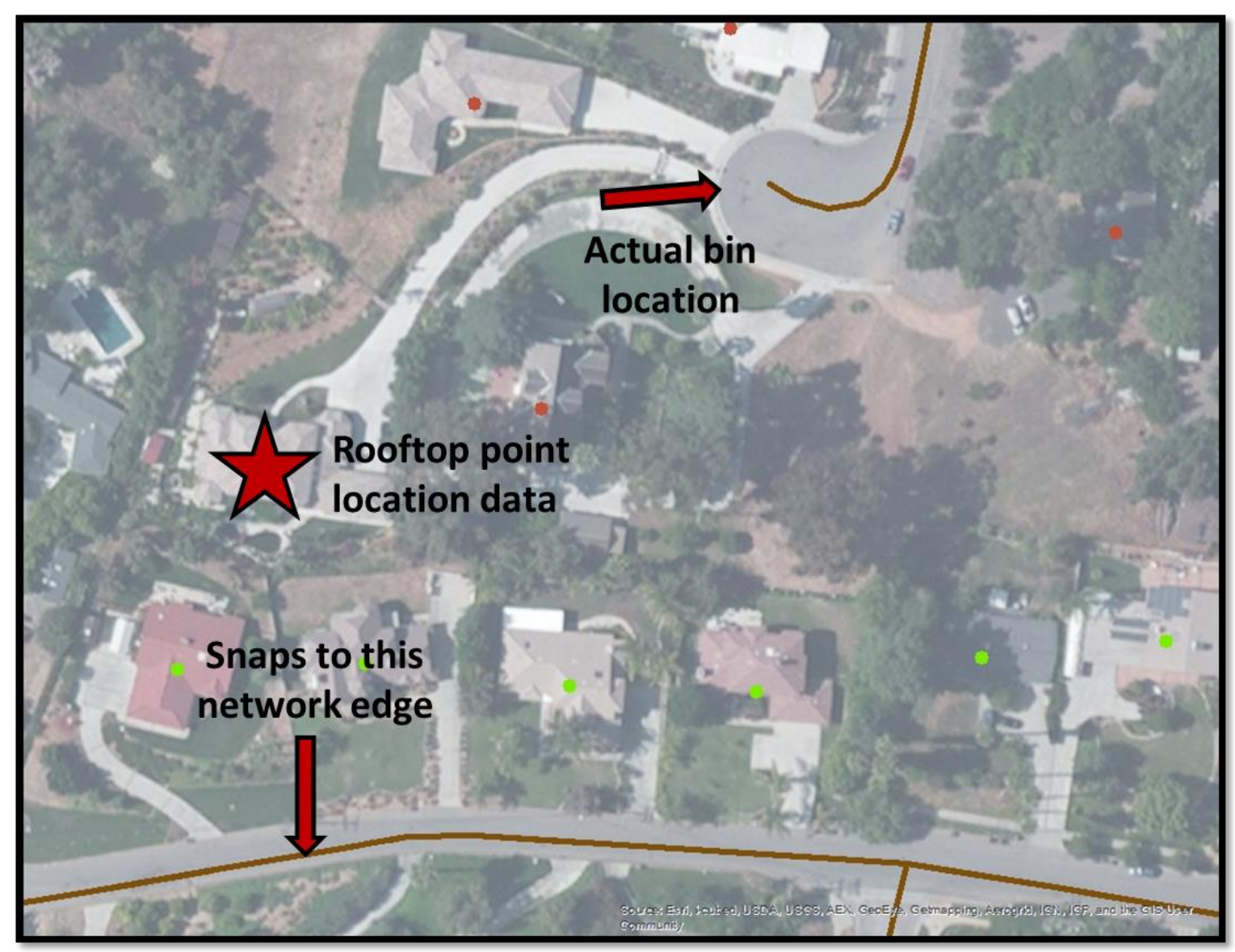

Figure -8: Incorrect Order Location along the Network Dataset

\subsubsection{Depots}

The VRP analysis layer uses depots for locations where a vehicle will start, unload, and end along a route on the network. This project used two depots. The start and end depots were point feature classes representing the sanitation truck yard located at 1270 West Park Avenue in Redlands (Figure 5-9). This is the location where the sanitation trucks are serviced and parked overnight. The depot properties adjusted were name, description, 
time window, and curb approach. The depot time windows denote hours the depot can be accessed during the workday; this property was set to 6:00 am to 2:00 pm. The curb approach property specifies direction the vehicle can arrive and depart from the depot. This property was set to either side of the vehicle, since there is no loading or unloading performed at this location. The second depot symbolizes the municipal landfill located at the northern end of Nevada Street (Figure 5-9). This point feature class represents where the location trucks will unload solid waste once they reach their 30 cubic yard capacity. This depot also used a time window of 6:00 am to 2:00 pm. Trucks can enter the landfill from any direction, so curb approach was set to either side of the vehicle. When sanitation trucks enter the city landfill, the rear bed is tilted upward to unload contents through the back end of the truck.

Figure 5-9: VRP Depots and Study Area

\subsubsection{Routes}

The route network analysis class identifies vehicle, driver, and route characteristics of the VRP. Route properties have many input settings that can be configured. This project used maximum number of orders, service time, overtime, start, end point location, and times. The max order count was set to 525 units. This value was the midpoint value of the suggested average truck capacity of 450 to 60096 -gallon bins. Two routes were constructed in order to serve the entire 829 collection points in the study area. Overtime was configured to compute whenever more than eight hours are required in a day. Start and end time fields were set for 6:00 am to 2:00 pm. This represents the standard workday for city sanitation employees. The municipal yard at 1270 West Park Avenue was set as the start depot. The city landfill located off North Nevada Street was set as the end depot. Service time was set for 10 minutes for both depot points. This value represents preparation time at the yard, and unloading time at the landfill. Once the VRP solver is run, a line feature traversing the network from the start depot to orders back to the end depot will be displayed. Additional output fields will also be automatically generated to include new values. The violated constraints field summarizes any operation violated or not performed. The first route had several unreachable orders on one street. The network Identify tool was used to manually detect the road edges missing connectivity. An edit session was used to connect edges and the network dataset was rebuilt. The solver was run to regenerate the routes to include previously unreachable orders.

\subsubsection{Breaks}

The breaks class is a non-spatial network analysis class that includes break periods for each route. Breaks are a standalone table, stored as a memory feature class in the analysis layer. The Quality of Life Department requires one 30 minute and two 15 minute breaks for drivers working a standard eight hour workday. These values were assigned to the routes and included in the breaks table. The time-window breaks were configured for 8:00 - 9:00 am, 10:00 am - 12:00 pm, and 12:00 - 1:00 pm; these represent the window of time each break can be taken. This configuration was used for all tests involving the 829 
orders. The city allows drivers to use the break periods at their own discretion. The IsPaid field is a Boolean value that determines if a break is paid or unpaid. The city pays the drivers during break time, so this value was set to true. After the solver was run, and the break table was populated with additional fields. These fields identify the sequence, distance, and time of each break along each route.

\subsubsection{Point Barriers}

Point barriers serve as added cost impedances in a network analysis. For this study, stop sign and traffic light point feature classes were used as added cost barriers. Traversing through the barrier increases the network cost by an assigned attribute value. The client supplied point feature class data representing the 1,290 stop signs and 94 traffic lights in Redlands (Figure 5-10). When point barriers are loaded, they are snapped to the nearest network edge to assure continuity. This continuity was established already within the network with the feature dataset topology rule. Stop signs were included in the network analysis layer as an added cost point barrier, with a 4-second cost delay. The CurbApproach value was set to (1) right side of vehicle. The CurbApproach property specifies the direction of traffic affected by the barrier. Traffic light points were loaded as an added cost barrier, with a 15-second cost delay. The CurbApproach value was set to (0) either side of vehicle. This cost barrier affects traffic travelling both directions along the network.

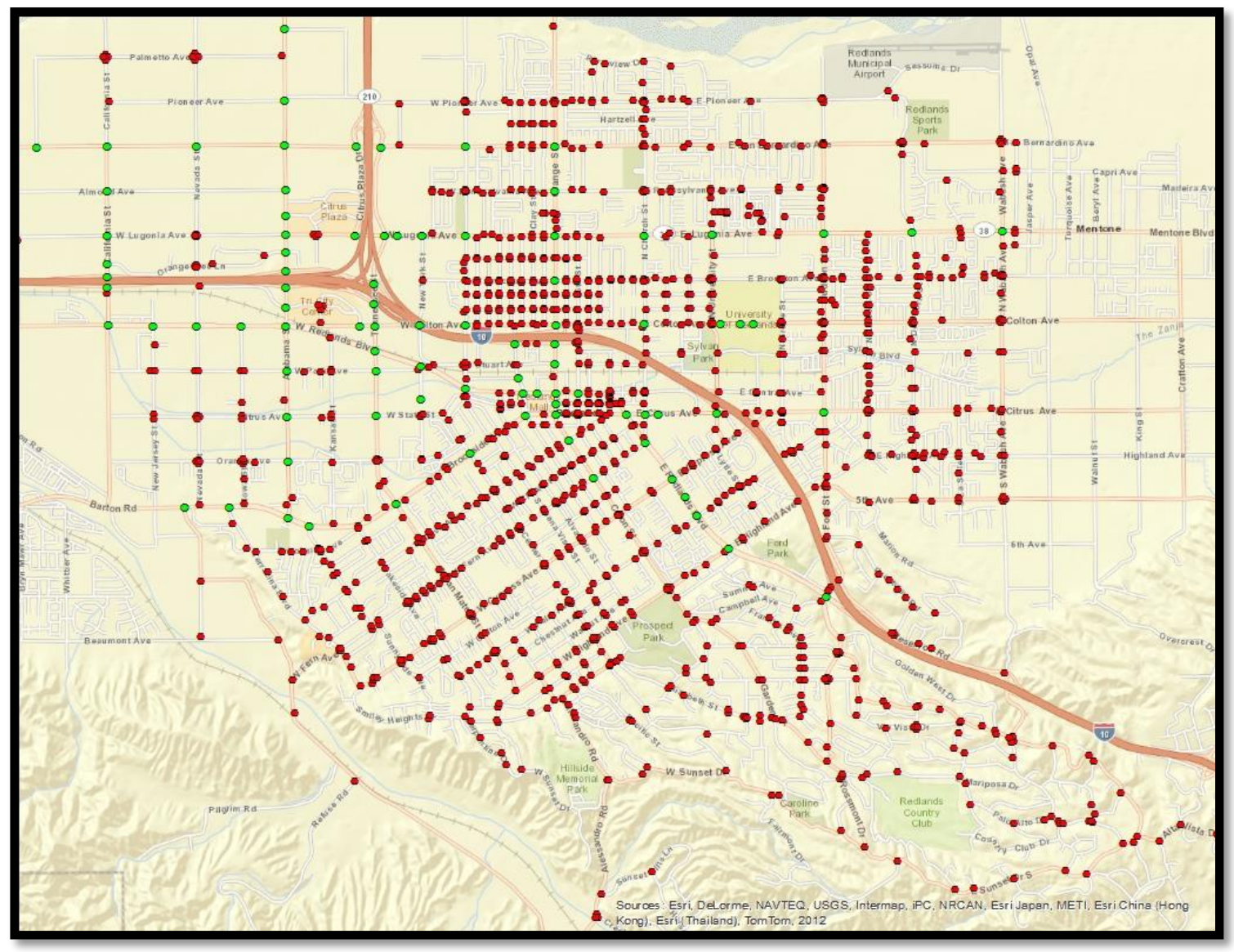


Figure -8: Added Cost Point Barriers

\title{
5.4 Executing the Vehicle Routing Problem Solver for New Routes
}

\author{
This section describes the steps used to configure and run the VRP solver without \\ any predefined constraints such as existing route, sequences, and \\ grouping. Different parameters and grouping were adjusted to \\ accommodate for anomalous results discovered after analysis. This \\ information is discussed in further detailed throughout Section $\mathbf{5 . 6}$ and \\ in Chapter 6. Once network dataset and analysis classes were built, and \\ the VRP analysis parameters were configured, parameters were set on \\ the layer properties dialog box for the analysis layer (Figure 5-11).
}

Figure 5-9: Network Analysis Vehicle Routing Problem Solver Layer Properties

The study was configured to solve for the best drive time. The DriveTime20 attribute field in the road feature class was used to calculate the drive times. The Distance Unit was set to miles. The U-turns at Junctions parameter was set to allow U-turns only at intersections and dead-ends. This setting was tested using other available choices, but did not produce practical results. After discussion with the client and field observation of the trucks on route, this was the closest setting to match actual driving behavior. True shape with measures was set for the Output Shape Type. This produced lines along each road segment of the output route and created a more user-friendly map for viewers following the route. The Restrictions Attribute for RestrictedRoads was selected here and allowed the solver to exclude certain roads along the network. Whenever the VRP solver is run the results are saved as a temporary layer within the Network Analyst window. It is a good practice to export the different layers to a permanent feature class format within the project geodatabase. This was done for: order, route, and break configuration, and retained the solver results data as attributes for each class.

\subsection{Configuring Existing Collection Routes for VRP Solver}

One of the objectives of the project was to compare new VRP generated collection routes to the existing routes. This allowed a direct comparison of total time and total miles driven between the different approaches for determining more efficient solid waste collection routes. To accomplish this, the existing route driven, sequence of waste bin collection, and grouping of orders had to be determined, replicated, and configured, then run through the VRP solver. Multiple meetings were conducted with city sanitation personnel to discuss and map current collection patterns. It was discovered that there are no standard collection patterns used; each driver has his own technique when driving and unloading bins within a collection district. Some drivers start from the outermost collection area boundary and work inward, either clockwise or counterclockwise. Some 
use a figure eight pattern to collect waste bins in a district. Others may only make U-turns at dead-ends, or intersections, or both. It was decided to use the route pattern from one city sanitation driver. After sketching his collection route onto a hardcopy map of the study area, this information was digitized into the project file geodatabase as a line feature class representing the current collection route. The next step was to assign a sequence attribute value to each collection point. This attempted to match the sequence in which waste bins are currently collected by the driver. This very tedious task involved an edit session on the collection points, then determining and manually inputting a sequence value for all 829 collection points. A new attribute field and values were input to identify which route the order was in. Since route order capacity was initially set to 525, two collection routes were identified to accommodate the 829 points. The VRP solver does not fully preserve an existing route and sequence for orders. To force the solver to use the existing route, a line feature was digitized around the entire existing route, and input into the VRP analysis layer as a line barrier. Restriction line barriers are feature classes in network analysis layers that can restrict or add cost to the underlying road edges of the network dataset. The restriction setting prohibits travel anywhere outside of the barrier, therefore forcing the solver to use the predefined existing routes within the restricted barrier boundary.

Once the two existing routes were input and configured in the solver, another change was made for the orders. Originally, the orders were set as a network analysis layer in the solver, and the Assignment Rule attribute field was adjusted to "override", which allows the solver to determine the route, grouping, and sequencing during calculation. To enforce the solver to only consider the existing route and sequence, the Assignment Rule was set to "preserve route and relative sequence". While the orders can be predefined to a specific route, the solver will only preserve the relative sequence for orders, not the absolute series compiled in the sequence attribute field. The settings of the other layers, including depots, routes, breaks, and point barriers, remain the same as previously described. Additional layers were added to the solver to reflect actual collection conditions. Depot layers were added from point feature classes to reflect the start and unload points. The breaks table was added to include the two 15 minute and one 30 minute break each driver takes during a standard eight hour workday. Added cost point barriers were included in the network analysis based on point feature classes that represent stop sign and traffic light impedances along the network. These barriers represent the 94 traffic lights and 1,290 stop signs along the streets of Redlands. All of these points have associated cost impedances assigned to them, lights having a 15 second and signs a four second time impedance value. When trying to model real travel conditions these values represent the average time a sanitation truck would spend at each location. These values were derived from discussions with city sanitation drivers, and Esri network specialists, as well as field observations. The VRP solver was then run to closely replicate the existing collection routes, grouping, and bin collection sequencing the city performs.

\subsection{Various Input Versions for VRP Solver}

Due to the anomalous results of solving all 829 collection orders at once, additional scenarios were tested until acceptable results were achieved. One of the attempted scenarios was to allocate the 829 collections to the two routes subjectively and then solve 
for each route individually. In doing so, the complexity of solving for two routes with over 800 points was reduced. This involved many of the same steps, starting with creating new analysis layers for each new route to be solved and then assigning many of the same parameters as before such as: importing a smaller group of orders, defining depots, identifying cost barriers, and assigning break periods. The break periods were now allocated differently; two breaks were assigned to one group and one break assigned the other group. The VRP layer properties analysis settings tab has an option to adjust how U-turns are addressed when traveling along the network. The U-turns at Junctions setting has four options available: allowed; allowed only at intersections and dead ends; allowed only at dead ends; and not allowed. The first three options were evaluated and tested on each route to evaluate how this setting impacted VRP solver performance and results. Because the advanced settings tab in the layer properties has an excess transit time setting, this was changed from the default medium to high. This setting attempts to further reduce transit time along the network. The time values for the global turn delay evaluator and added cost impedances were adjusted to see how this impacts total travel time results. The testing results of different scenarios and parameter settings will be detailed in Chapter 6.

\subsection{Network Analyst Route Solver}

Esri's ArcGIS Network Analyst extension also has a Route Solver. This tool is a simpler version of the Vehicle Routing Problem solver, but performs similar functions. The Route Solver is used to find the quickest or shortest route between two or more points on a network. The Route Solver can factor in impedances during analysis; the difference between the two solvers is that the VRP solver can solve multiple routes with more parameter settings such as breaks, while the Route Solver only works with a single route. The Route Solver can reorder stops to find the optimal route, which is known as the Travelling Salesman Problem (TSP). Although this project focused on evaluating the VRP solver for solid waste collection, some tests were done using the Route Solver to gauge its functionality. This section explains the process to configure and execute the Route Solver for solid waste collection optimization.

A new Route Solver analysis layer was created to store network classes used during route analysis. The Route Solver uses stops instead of orders to identify collection locations along the route. The Route Solver does not use depot locations to identify start and finish points along the network. To accomplish this, municipal yard and landfill point features were included with the stops and identified as the first and last stop. This was accomplished by enabling the setting of "preserve first and last stop" in the Route Solver layer properties to preserve first and last stop; this was enabled. The U-turns policy was set to allow at intersections and dead ends. The Route Solver was configured and run to calculate shortest distance and quickest travel time. These results are discussed in Chapter 6.

\subsection{Summary}

This chapter discussed the components, configuration, and implementation of the VRP solver for this project. The experiment for calculating slope and incorporating the results into a network dataset was reviewed. This project involved multiple configurations and 
collection scenarios that were run through the VRP solver. The Network Analyst Route solver was also introduced and discussed as an option to use in performing route optimization. 


\section{Chapter 6 - Results and Analysis}

This chapter discusses the overall results of the project and how the client might benefit from the information. The results from configuring and running the Network Analyst Vehicle Routing Problem solver for new and existing routes are reviewed.

\subsection{New Routes from the Network Analyst VRP Solver}

The first scenario run through the VRP solver involved importing all 829 orders and letting the solver determine allocating all the orders into two routes and collection sequence. The version with "U-turns allowed at intersections and dead ends" setting seemed to produce better results than having it set to "allow only at dead ends". However, the total travel time was much higher than expected in both cases. The total traveltime from "U turns allowed at intersections and dead ends" was 635 minutes, which equates to 10 hours 35 minutes, more than two hours beyond what was expected. Allowing U-turns only at dead ends produced slightly higher results. Upon reviewing the results for any scenarios with all 829 points, the orders displayed very odd sequencing values for each collection point. Some areas had consecutive sequencing values, while most others had sporadic number sequences and a truck would need to constantly travel 
back and forth to service orders. Because the trucks collect waste bins from the right side of the vehicle, point collection sequencing must reflect that. Figure 6-1 is an example of one area with anomalous sequencing results. The anomalous sequencing values explained why the total time solved was considerably higher than expected.

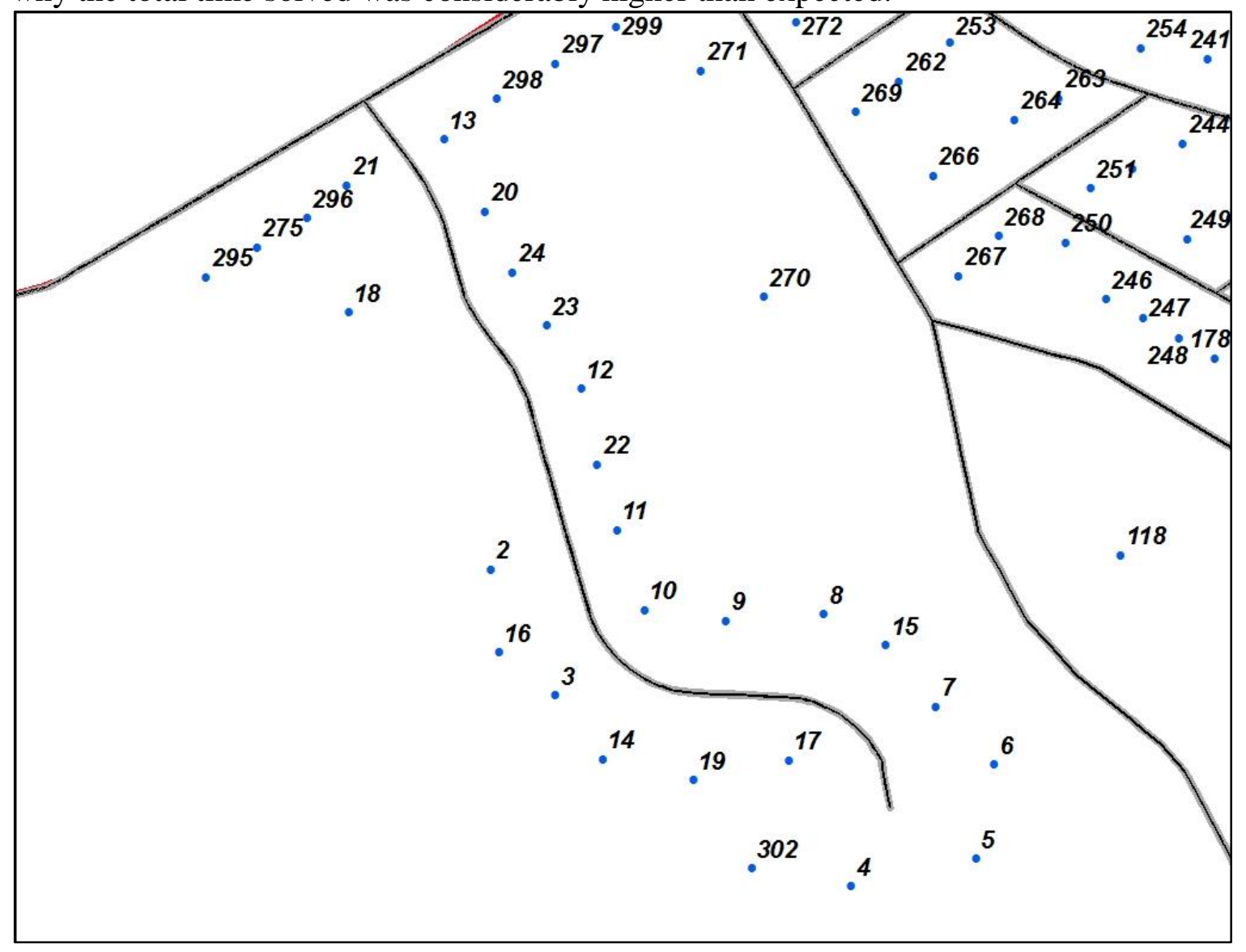

\section{Figure -1: Anomalous Sequencing Results}

The next scenario involved solving the 829 points as two separate groups. The same parameters were configured except the two groups were predefined to 381 points for Route 1 and 448 points for Route 2 . The sum of travel time from these two routes was considerably lower, with a total time of $423 \mathrm{~min}$. (7 hrs. $23 \mathrm{~min}$.). The anomalous sequencing was not present in VRP Route 2 but did exist in VRP Route 1. This was still unacceptable, so another solve routine was performed using the same two predefined groups of 381 and 448 points. This time they were solved together in one solve routine, but the AssignmentRule setting on the orders was changed to "preserve route". This setting forced the VRP solver to include the 381 orders on Route 1 and the 448 orders on Route 2. The total time was $544 \mathrm{~min}$ (9 hrs. $4 \mathrm{~min}$.). This was lower than the original result of $635 \mathrm{~min}$., but higher than individually solved results of $423 \mathrm{~min}$. The third test result still showed anomalous sequencing values throughout both collection routes. Table 6-1 shows results using the Network Analyst VRP solver to serve 829 order points. 
Table 6-1. Results Comparison Solving All 829 Collection Points

\begin{tabular}{|c|c|c|c|c|c|c|c|c|c|}
\hline \multicolumn{7}{|c|}{ VRP Determined Route, Group, \& Sequence } & \multicolumn{3}{|c|}{ Predefined Route, Group, \& Sequence } \\
\hline & VRP Rt 1 & VRP Rt 2 & VRP Total & VRP Rt 1 & VRP Rt 2 & VRP Total & VRP Rt 1 & VRP Rt 2 & VRP Total \\
\hline Bins & 525 & 304 & 829 & 381 & 448 & 829 & 381 & 448 & 829 \\
\hline Miles & 51 & 95 & 146 & 28 & 33 & 61 & 72 & 76 & 148 \\
\hline \multirow[t]{2}{*}{ Time (min) } & 265 & 370 & 635 & 265 & 158 & 423 & 270 & 274 & 544 \\
\hline & \multicolumn{3}{|c|}{ Solved as one } & \multicolumn{3}{|c|}{ Solved Individually } & \multicolumn{3}{|c|}{ Solved as one } \\
\hline
\end{tabular}

\begin{tabular}{|c|c|c|c|c|c|c|}
\hline \multicolumn{7}{|c|}{ Predefined Grouping Comparison } \\
\hline & \multicolumn{3}{|c|}{ Predefined Grouping Sequencing } & \multicolumn{3}{|c|}{ Predefined Grouping Sequencing } \\
\hline & Existing Rt 1 & Existing Rt 2 & Existing Rt Total & Existing Rt 1 & Existing Rt 2 & Existing Rt Total \\
\hline Bins & 456 & 364 & 820 & 522 & 294 & 816 \\
\hline Miles & 38 & 38 & 76 & 34 & 29 & 63 \\
\hline Time (min) & 171 & 190 & 361 & 265 & 370 & 635 \\
\hline & \multicolumn{3}{|c|}{ Solved Individually } & \multicolumn{3}{|c|}{ Solved as one } \\
\hline
\end{tabular}

Additional VRP solver configurations were conducted to identify the cause of the anomalous sequencing of orders. A selection of 168 arbitrary points within the study area produced the same results of uncharacteristic order sequencing along the two routes. Esri Network Analyst professionals recommended much smaller groups for orders, so a subset of 87 arbitrary points was selected and tested. Similar configurations to earlier studies were arranged in the VRP solver. The first test was solving for 87 orders for two separate groups. The route maxordercount parameter was set to 45 orders; this tells the VRP solver once 45 orders are collected on route 1 , the truck travels to the landfill depot to unload. The truck starts route 2 from the landfill depot, serving the remaining 42 orders, then returns to the landfill depot for a final unloading. This produced acceptable results with no anomalies in sequence values. Another test conducted was predefining the group and waste bin sequence to serve the 87 orders. This was achieved by assigning the same 45 and 42 points to route 1 and 2 respectively. This would match the grouping from the first test. A logical collection sequence was then determined and manually input to the waste bin attributes of each order group. This would attempt to match actual waste bin collection patterns a city sanitation driver may use. Before the solver was run, the order assignment rule attribute was changed to "preserve route and relative sequence". Once the VRP solver was run, the results were as expected; the sequencing and grouping were maintained. 
Table 6-2. Results Comparison Solving 87 Collection Points

\begin{tabular}{|c|c|c|c|c|c|c|}
\hline & \multicolumn{3}{|c|}{ VRP Determined } & \multicolumn{3}{c|}{ Predefined Grouping } \\
\hline & VRP Rt 1 & VRP Rt2 & VRP Total & Defined Rt 1 & Defined Rt 2 & Defined Total \\
\hline Bins & 42 & 45 & 87 & 42 & 45 & 87 \\
Miles & 11 & 19 & 30 & 14 & 19 & 33 \\
Time (min) & 63 & 101 & 164 & 76 & 101 & 177 \\
\hline & \multicolumn{3}{|c|}{ Solved as one } \\
\hline
\end{tabular}

\begin{tabular}{|c|c|c|c|c|c|}
\hline & \multicolumn{5}{|c|}{ Predefined Group \& Sequencing } \\
\cline { 2 - 6 } & Defined Rt 1 & Defined Rt 2 & Defined Total & Defined Rt 1 & VRP Rt 1 \\
\hline Bins & 42 & 45 & 87 & 87 & 87 \\
Miles & 11 & 19 & 30 & 17 & 17 \\
Time (min) & 64 & 71 & 135 & 90 & 90 \\
\hline & \multicolumn{5}{|c|}{ Solved as one } \\
\hline
\end{tabular}

The results from both tested are summarized in Table 6-2. The VRP solver produced a route with a total travel time of $164 \mathrm{~min}$. while the predefined route was $177 \mathrm{~min}$. This showed a 7\% improvement using the VRP route compared to a route with just a predefined group. When using a predefined group and collection sequence, the result was 135 min. showing a $17 \%$ improvement. Solving all 87 orders at once with one route produced the same 90 min. result for both VRP and predefined versions.

\subsection{Existing Routes from Network Analyst VRP Solver}

This section reviews the results from running the VRP solver with predefined constraints, attempting to replicate existing collection routes travelled. As discussed in Section 5.5, a collection route and pattern were input into GIS format for inclusion in the VRP solver. The tedious task of manually digitizing existing routes and 829 waste bin collection sequences was completed in order to replicate actual pickup patterns. Most analysis parameters were configured the same as earlier tests, except for the assignment rule for the orders. This attribute field values were changed to "preserve route and relative sequence" then solved. This forced the VRP solver to try to preserve existing route and collection sequence for orders. Only the relative sequence was maintained, not the absolute sequence. This test solved for two routes individually: one with 456 orders and 
the other with 373 . Figure 6-2 depicts the orders for existing route 1 with sequence values shown as labels.

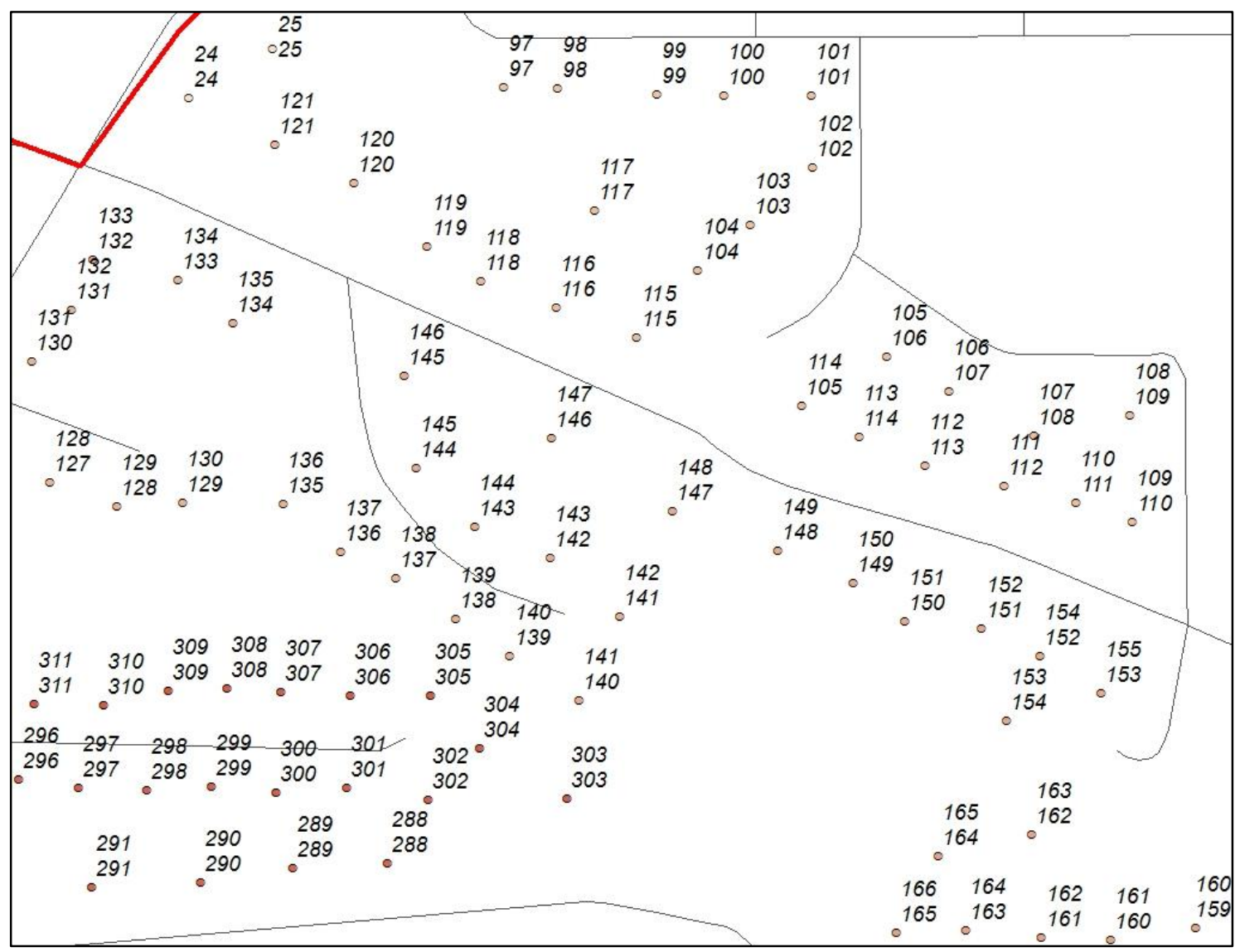

\section{Figure -2: Existing Route Collection Sequence Comparison}

The top value is the sequence number the VRP solver produced; the bottom number is the predefined sequence value that matches existing waste bin collection patterns. There are a total of 456 orders on this route; the VRP solver preserved the predefined sequence order for the majority of the points. If there were differences in values it was only by one digit, which may be due to minor typographic errors with the manual input sequence of all 829 points. The Network Analyst VRP solver did a decent job preserving the predefined grouping, sequencing, and routes to match existing collection conditions for both routes. Anothertest was conducted using predefined route, grouping, and sequencing for all collection points with the VRP solving both routes in one solve routine, rather than individually.

All the parameters were similar to earlier configurations except for maxordercount, which was set to 522 for route 1 and 294 for route 2 . A line barrier restriction feature class was used around the existing routes to prevent the VRP solver from calculating a 
new route. . The results for both routes were a total travel time of time of $635 \mathrm{~min}$. (10 hrs. 35 min.). The solver did a very good job maintaining the exact predefined collection sequence values, but the total travel time was well beyond what was expected when trying to match actual collection conditions.

The processing times for the VRP solver varied considerably between the different scenarios tested. When solving all 829 orders in one solve routine with no predefined constraints, the processing time was around 2 hours. However, the processing time was reduced to a few minutes when solving all 829 points in one solve routine with predefined constraints for route, grouping, and sequencing.

\subsection{Results from Network Analyst Route Solver}

The Network Analyst Route solver was used to conduct a route optimization test on 87 points in the project study area. This solver was used to calculate shortest distance and quickest routes for the smaller group study area. The VRP solver only solves for quickest time to travel a route, the Route Solver provides an option to solve for shortest distance. The Route Solver is a simplified version of the Vehicle Routing Problem solver and does not have options for break periods, service times, enhanced time windows, or depot locations. When solving for shortest distance, the route solver calculated 28 miles in 73 minutes for two routes to stop at the 87 points. Solving for quickest travel time calculated 29 miles in 66 minutes for two routes to stop at 87 points. The Route solver produced the expected results and the processing time was very quick, under one minute for each test. Table 6-3 shows the Route solver results. The travel time results from the Route Solver should not be compared to the VRP solver results since there are fewer parameters and time attributes configured in the Route Solver.

Table 6-3. Results from Network Analyst Route Solver

\begin{tabular}{|c|c|c|c|ccc|}
\hline & RS Rt 1 & RS Rt2 & RS Total & RS Rt 1 & RS Rt2 & RS Total \\
\hline Bins & 42 & 45 & 87 & 42 & 45 & 87 \\
Miles & 10 & 18 & 28 & 11 & 18 & 29 \\
Time (min) & 26 & 47 & 73 & 24 & 42 & 66 \\
\hline & \multicolumn{3}{|c|}{ Solved for Distance } & \multicolumn{3}{c|}{ Solved for Time } \\
\hline
\end{tabular}

\subsection{How the City of Redlands Might Use the Results}

The City of Redlands wanted to see how GIS technology could be used to help reduce costs for solid waste collection and transportation. The results from this project provide a starting point and guideline when using Esri Network Analyst Vehicle Routing Problem solver software version 10.1 to perform route optimization for solid waste collection and transport. This project identifies VRP solver configurations that work well and ones that do not work well. The VRP solver seems better suited for orders of 150 or less. The 
solver does not produce optimal routing scenarios when orders are in the hundreds and have multiple routes to calculate at once. When solving for time as a cost, the solver consistently produced anomalous results in the sequencing to collect orders, which contributed to longer than expected travel times.

The accuracy level and integrity of the data input to the VRP solver greatly impacts results and overall editing time and effort. When performing route optimization using Esri's Network Analyst extension, having an accurate, fully contiguous network dataset is imperative. This project produced an updated and edited network dataset of all roads within the City of Redlands. This project used rooftop point location data to represent the residential waste bins to service within the project study area. These data were edited to better represent actual bin locations for homes with longer driveways. Certain rooftop data locations with longer driveways had a tendency to match to incorrect street edges along the network. This presented a problem during analysis and contributed to extended amounts of editing time. These data could be used again by the city, but a more accurate location of waste bins being collected would be ideal. GPS positional data for each bin location and collection sequence would help produce more accurate results and considerably reduce editing times. The project used sketches to reproduce existing collection routes driven. This was time consuming and had accuracy limitations. If the city continues using the VRP solver for optimization, any available GPS data for current collection routes driven should be evaluated for use.

The City of Redlands could use this project as a guideline to configure Esri's VRP or Route solvers for solid waste collection. This project explains how the Network Analyst VRP solver is not intended for analysis of hundreds of orders at a time. The city could start by using the VRP solver to analyze smaller groups of orders, such as commercial dumpster collections. The Network Analyst Route solver was also identified as a simpler versioned GIS route optimization tool to be used. There are commercial routing software packages available, such as RouteSmart, which focus on route optimization for high density orders. 


\section{Chapter 7 - Conclusions and Future Work}

This project set out to evaluate how GIS technology could be used to help reduce overall costs for collection and transportation of residential solid waste for Redlands, California. The city has a functioning GIS department that will assess and implement some of the projects findings. The project focused on using Esri's Network Analyst software extension and its route optimization solvers. The primary solver evaluated was the Vehicle Routing Problem solver. This route optimization tool was introduced in ArcGIS version 9.3 and is designed to calculate quickest collection or delivery travel times for a fleet of vehicles. The Network Analyst Route solver was also evaluated. This is a simpler version of the VRP solver and does not have as many parameters to configure, but allows users to solve for shortest travel distance. This chapter also discusses the future work that can be done from this project.

The City of Redlands has GPS units on each of the 28 sanitation trucks. The GPS data provides city managers a reference to vehicle location and driving patterns. Several attempts to obtain the vehicle GPS data were made but were unsuccessful.

\subsection{Conclusions}

This project evaluated Esri's Network Analyst software extension to see how different solvers could help reduce costs for sanitation collection and transportation. The first tests were done using the Vehicle Routing Problem solver. This solver is intended for route optimization of fleet vehicles and allows users to configure different parameters to match actual travel conditions. A small study area was chosen in the south part of Redlands, one sanitation district for collection of residential solid waste from homes. The collection locations were derived from rooftop point data supplied by the city, representing 829 homes with waste bins placed curbside for collection. Several meetings were conducted with City of Redlands GIS and sanitation personnel to gain a better understanding of project expectations, as well as to obtain knowledge of sanitation collection planning and processes. Different route optimization software packages were researched, and the decision was made to evaluate Esri's Network Analyst solvers. The City of Redlands GIS department maintains licensing for ArcGIS software which includes the Network Analyst extension and will be incorporating data and findings into its GIS. The VRP solver is intended to solve for quickest time travelling point-to-point along a defined network. A network dataset was constructed to represent all streets within the City of Redlands. This

originated from a line feature class of roads with corresponding attribute information also used during route analysis. The VRP solver incorporates cost impedance values on the network that are accumulated during travel. Some cost impedances were: travel time, stop signs, and traffic lights. The software evaluates the costs and determines the least cost path to travel. Different parameters were input to match collection conditions such as: break periods, time windows, service times, driving pattern, and side of curb. After configuration, the VRP solver was run to determine new routes to service 829 collection points inside the study area. The VRP solver did not return reasonable results when solving for large groups of points, typically amounts over 150 orders. The solver returned results showing illogical sequencing for points being serviced, which contributed to longer than expected travel times. Different configurations and quantities were tested 
until an acceptable result was achieved. A final random group of 87 points within the study area produced improved results.

Similar tests were conducted using ArcGIS Network Analyst Route solver. This solver can solve for quickest time or shortest distance, but has limited parameters for matching actual travel conditions. Test results using Route Solver with 87 collection points produced sufficient results, as expected. The City of Redlands will be able to refer to this project when implementing a GIS for route optimization in collecting and transporting solid waste.

\subsection{Future Work}

This project provides a starting point for future work using ArcGIS Network Analyst solvers for route optimization. The results of this project show the VRP solver is not ideal for calculating route optimization for large clusters of points. One option for future work is that the city could use the project's network dataset with the VRP solver to calculate route optimization for commercial dumpster waste collection. These are considerably smaller clusters of points and would be spread throughout the city. The VRP solver successfully reduced cost and travel time for a group of 87 points when compared to existing routes traveled. If the city decides to solve larger clusters of points representing residential customers, one workaround might be to include only one order along the midpoint of each road segment, with this one point representing the multiple orders along each road network segment. The ArcGIS geoprocessing tool feature to point creates a point feature class from representative locations of input features. Consolidating residential orders to service into groups of 150 or less should produce more accurate results from the VRP solver. Future work could be to use this approach to construct optimized routes for collecting large bulk items from residents. These may be smaller groups of prescheduled pickups for oversized items that sanitation trucks could not pick up. These items tend to be furniture, large appliances, debris, and construction material. Once a successful solver configuration is assembled, several steps could be incorporated into ArcGIS Model Builder to automate the procedures. Another enhancement to this project could be expanding the existing network dataset into a 3D dataset. This involves calibrating network edges with directional impedance attributes for the distance travelling uphill. Calculating slope values for each road segment would not be enough. Since slopes are directional, a bi-directional attribute would need to be created for each segment. Once constructed, these attribute values could be used as additional cost impedances for modeling actual travel conditions.

The City of Redlands provides many different services to residents, and GIS technology can be used to help improve performance and reduce costs. This project could be used as a guideline for configuring route optimization for any services that travel within the city to perform a pickup or delivery. This could be school bus, public transportation routes, street sweeping, scheduled utility asset management and maintenance, or any other city service that has multiple stops along the network. 


\section{Works Cited}

Aguilar, S. (2000). City of Los Angeles Applies ArcLogistics Route. ArcNews.

Cannon, J. S. (2005). Inform, Inc. Retrieved October 2, 2012, from Inform, Inc.: http://www.informinc.org

CensusViewer. (2013). Redlands, California Population: Census 2010 and 2000 Interactive Map, Demographics, Statistics, Quick Facts. Retrieved from http://censusviewer.com/city/CA/Redlands.

Chalkias, C., \& Lasaridi, K. (2011, August 23). Benefits from GIS Based Modelling for Municipal Solid Waste Management. (M. S. Kumar, Ed.) Integrated Waste Management, I, 417-436.

Esri. (2012). Vehicle Routing Problem Analysis. Retrieved May 2013, from Esri ArcGIS 10.1 Help: www.esri.com

Jovicic, N. M., Boskovic, G. B., Vujic, G. V., Jovicic, G. R., Despotovoc, M. Z., Milovanovic, D. M., et al. (2011). Route Optimization to Increase Energy Efficiency and Reduce Fuel Consumption of Communal Vehicles. Thermal Science, 14, S67-S78.

Karadimas, N. V., Doukas, N., Kolokathi, M., \& Defteraiou, G. (2008, December). Routing Optimization Heuristics Algorithms for Urban Solid Waste Transportation Management. WSEAS Transactions on Computers, 7(12), 20222031.

Karadimas, N. V., Kolokathi, M., Defteraiou, G., \& Loumas, V. (2007). Municipal Waste Collection of Large Items Optimized with Arc GIS Network Analyst. 21st European Conference on Modelling and Simulation

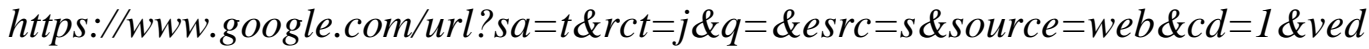
$=0 C C O Q F j A A \& u r l=h t t p \% 3 A \% 2 F \% 2 F w w w$. researchgate.net $\% 2 F$ publication $\% 2$ F229007213_MUNICIPAL_WASTE_COLLECTION_OF_LARGE_ITEMS_OPTI MIZED_WITH_ARC.

Lovell, J. (2007, December 9). Lefthand Turn Elimination. The New York Times. Retrieved July 15, 2013, from http://www.nytimes.com/2007/12/09/magazine/09left-handturn.html

Modak, E. A. (1996). Optimal Regional Scheduling of Solid Waste System. II: Model Solution. ASCE Journal of Environmental Engineering, 793-799.

Sandhu, J. (2011). Calibrating a Transportation Network with Terrain Information for Routing Applications. Applied Geography Conference. Esri, Inc.

Spector, D. (2013, March 15). Why Left-Hand Turns are Burning a Hole in Your Wallet. Retrieved July 15, 2013, from Business Insider: http://www.businessinsider.com/save-money-gas-avoid-left-turns-2012-3

Tavares, G., Zsigraiova, Z., Semiao, V., \& Graca Carvalho, M. (2008). A Case Study of Fuel Savings Through Optimisation of MSW Transportation Routes. Management of Environmental Quality: An International Journal, 19(4), 444-454. 

